\title{
PRÁCTICAS DE MANUFACTURA ALFARERA DE LAS COMUNIDADES ALDEANAS DE INICIOS DEL PRIMER MILENIO (SIGLOS III-VI DC) EN EL NOROESTE ARGENTINO. ESTUDIOS DE PASTAS CERÁMICAS DE UCHUQUITA (ANILLACO, LA RIOJA)
}

\author{
POTTERY MANUFACTURING PRACTICES OF THE VILLAGE COMMUNITIES \\ OF THE BEGINNINGS OF THE FIRST MILLENNIUM (CENTURIES III-VI \\ DC) IN THE ARGENTINE NORTHWEST. STUDIES OF CERAMIC PASTES OF \\ UCHUQUITA (ANILLACO, LA RIOJA)
}

Sebastián Andrés Carosio ${ }^{1,2,3}$, Gabriela Sabatini ${ }^{4}$ y Pablo Andrés Cahiza ${ }^{3}$

\begin{abstract}
En este artículo se presentan los resultados obtenidos del estudio de cerámicas procedentes del conjunto arquitectónico Uchuquita, ubicado al norte de la Sierra de Velasco, Noroeste argentino (Anillaco, La Rioja, Argentina). Esta zona fue ocupada durante los inicios del primer milenio de la era (siglos III-VI DC) y constituye una de las primeras evidencias de las comunidades aldeanas que habitaron la región. A partir de un análisis submacroscópico y microscópico de pastas se reconocen aspectos composicionales y su relación con el perfil geológico del área, y las elecciones técnicas dentro del proceso de producción alfarera. La información obtenida permite proponer la existencia de una producción local/microrregional para la mayoría de los bienes alfareros consumidos en el área, así como diversidades y uniformidades tecnológicas en diferentes fases de la cadena operativa. Además, se plantea la existencia de una tradición alfarera basada en la cotidianeidad de la práctica, el ambiente, la circulación de bienes y personas, y el mantenimiento de vínculos socioculturales y tecnológicos regionales, en un contexto de producción doméstica, de baja escala. Los datos aportan al conocimiento de las trayectorias históricas de las comunidades locales y son una contribución empírica comparativa para esta región de los Andes.
\end{abstract}

Palabras claves: alfarería, petrografía, primer milenio, comunidades aldeanas, Noroeste argentino.

This article presents the results from the study of ceramics from the Uchuquita architectural complex, located north of the Sierra de Velasco, Argentine Northwest (Anillaco, La Rioja, Argentina). The area was occupied during the beginning of the first millennium of the era (III-VI centuries $A D$ ) and constitutes one of the first evidence of the village communities that inhabited the region. From a submacroscopic and microscopic analysis of pastes, we recognize compositional features and their relationship with the geological profile of the area, as well as the technical choices during the pottery production process. The information obtained suggests the existence of local/ microregional production for most of the pottery goods consumed in the area, as well as technological diversities and uniformities in different phases of the operational chain. Likewise, we consider the existence of a tradition of pottery based on the daily practices, the environment, the circulation of goods and people, and the maintenance of regional socio-cultural and technological links, in a context of low-scale domestic production. The data contribute to the knowledge of the historical trajectories of the local communities and are a comparative empirical contribution for this region of the Andes.

Key words: Pottery, petrography, first millennium, village communities, Argentine Northwest.

El objetivo de este estudio es contribuir al conocimiento sobre la tecnología y prácticas de manufactura alfarera de los ceramistas que habitaron el conjunto arquitectónico
Uchuquita, ubicado en las cercanías de la localidad de Anillaco, en el faldeo oriental de la Sierra de Velasco (La Rioja), Noroeste argentino (NOA), durante el inicio del

\footnotetext{
${ }^{1}$ CONICET Consejo Nacional de Investigaciones Científicas y Técnicas, Buenos Aires, Argentina.

${ }^{2}$ Departamento de Geología, Facultad de Ciencias Físicas, Matemáticas y Naturales, Universidad Nacional de San Luis, San Luis, Argentina. sebacarosio@yahoo.com.ar

${ }^{3}$ Instituto de Arqueología y Etnología, Facultad de Filosofía y Letras, Universidad Nacional de Cuyo, Mendoza, Argentina. pcahiza@mendoza-conicet.gob.ar

${ }^{4}$ Centro Regional de Investigaciones Científicas y Transferencia Tecnológica de Anillaco, La Rioja (CRILAR), La Rioja, Argentina.gsabatini@crilar-conicet.gob.ar
} 
primer milenio (ca. siglos III-VI DC). A partir de análisis submacroscópicos y microscópicos de pastas correspondientes a diferentes conjuntos cerámicos, se busca identificar la composición de las materias primas elegidas por los alfareros, así como a las decisiones técnicas dentro del proceso de fabricación, como un medio para indagar sobre las tradiciones tecnológicas y socioculturales de los grupos que habitaron el área.

Se concibe a la tecnología cerámica como el proceso y resultado de una "cadena operativa" (LeroiGourhan 1964), la cual depende y deriva de una serie de elecciones, conocimientos y gestos, conscientes o no, durante cada una de las etapas de fabricación. En ella se entrelazan las habilidades, funcionalidades, relaciones sociales y modelos de enseñanza/aprendizaje de los ceramistas. Además, representa el desarrollo de pautas culturales materializadas, inmersas en un contexto temporal y espacial particular (Dobres y Hoffman 1994; Miller 2007; Rye 1981). Se entiende que todas las acciones en la cadena operativa son plasmadas en un estilo tecnológico, un conjunto de "formas de hacer" en el que diferentes atributos (pastas, técnicas de levantado, formas, decoraciones y cocciones) son regulares y habituales, definidos en un momento histórico, y expresan, aunque no necesariamente de manera directa, identidad social o un proceso mediante el cual esta es formada y transformada. Un estilo tecnológico muchas veces incluye modalidades internas de manufactura, asociadas a leves variaciones en cualquiera de los atributos, pero tanto los puntos de diferencias como los de semejanzas deben confluir en un patrón común que responde a una misma pauta de racionalidad (Lechtman 1977; Lemonnier 1992; Sanhuesa 2006; Stark 1999).

La cadena operativa cerámica se encuentra sujeta a decisiones habituales de elaboración, uso, intercambio y abandono de las vasijas en un espacio social. Esto le otorga un importante potencial para descifrar los modos de producción y su contexto en las sociedades del pasado (Shanks y Tilley 1987). Cada elección técnica dependerá de múltiples factores, tales como la necesidad mecánica y/o funcional del recipiente a elaborar, la disponibilidad de materias primas que exhibe el ambiente, valores sociales e identitarios e influencias externas, entre otros. En cualquier caso, cuando las prácticas son reproducidas y mantenidas en el tiempo, conforman una tradición, y son inherentes a las dimensiones políticas y económicas en que se enmarcan (Dobres y Hoffman 1994; Gosselain 1992; Sillar 2009).

Las primeras fases dentro de la cadena operativa cerámica incluyen la selección de materias primas y su tratamiento para la obtención de la pasta, mezclando de manera consciente e inconsciente aquellos materiales que van a configurar la estructura del recipiente (Olaetxea
2000). Desde el punto de vista técnico y social, la comprensión de esta etapa posee gran significancia, ya que representa un ámbito de la producción generalmente poco visible, reservado para los productores y no los consumidores (Albero Santacreu 2014; Calvo Trías y García Rosselló 2011). Entre los estudios de pastas cerámicas se encuentran los análisis petrográficos, practicados a partir de la observación microscópica de láminas delgadas de tiestos (Ghezzi 2011; Gibson y Woods 1990; Shepard 1976 [1956]; entre otros). Ellos permiten identificar y cuantificar los minerales, rocas y otros componentes presentes en las pastas, así como -aunque no siempre es posible- discriminar entre aquellos materiales propios de las arcillas y los agregados por los alfareros. Además posibilitan acercarse a los procedimientos aplicados en la preparación de las materias primas, en el levantado, y en la cocción. También permiten conocer atributos funcionales y mecánicos, y aspectos de carácter sociocultural en el proceso de manufactura. Finalmente, brindan información sobre a la procedencia geológica de materias primas, aunque debe complementarse con exámenes químicos para obtener resultados certeros (Livingstone Smith 2007; Middleton et al. 1985; Neff 1992).

\section{Antecedentes}

Desde inicios de siglo XX hasta la década de 1980 los trabajos arqueológicos en Anillaco se redujeron a hallazgos aislados producto de expediciones y relevamientos en diferentes zonas del centro norte de La Rioja (Alanis 1974; Boman 1927-1932; Cáceres Freire 1937). Posteriormente comenzaron los trabajos sistemáticos (Dlugosz et al. 2009; Mercado 1994; Ortiz Malmierca 2001; Raviña y Callegari 1992), abarcando el estudio del patrón de asentamiento, la arquitectura y otros restos materiales, y las primeras dataciones radiocarbónicas. Las investigaciones revelaron diversas unidades arquitectónicas distribuidas en cada una de las cuencas del faldeo oriental de la Sierra de Velasco, generalmente compuestas de patios y recintos pircados de paredes dobles y simples, forma cuadrangular y rectangular, además de campos de terrazas de cultivo distribuidos entre los sitios, construidos con paredes de piedra de escasa altura y morteros tallados en grandes rocas. Estos asentamientos darían cuenta de las primeras ocupaciones aldeanas estables y económicamente productivas del área.

En los últimos años avanzaron las investigaciones en Uchuquita (Anillaco) y en las cuencas cercanas, incluyendo prospecciones de cobertura total, análisis espaciales, excavaciones, nuevas dataciones y el estudio de materiales arqueológicos (Cahiza 2015; Cahiza et al. 2017; Sabatini y Garate 2017; Sabatini y Salminci 2016). 
Los resultados sostienen que en el área se extendieron comunidades aldeanas con diferentes estrategias de aprovechamiento y transformación del espacio natural y cultural. Este proceso habría poseído al menos tres temporalidades: en primer lugar se habrían desarrollado, entre los siglos III y VII de la era, grupos sedentarios de familias con estructura descentralizada y escasa desigualdad social. Hacia el siglo VII las comunidades conformarían unidades aldeanas de mayor tamaño asociadas a arquitectura de uso público y a espacios de producción agrícola que indicarían una intensificación productiva, integración residencial e interacción regional. Finalmente, hacia fines del primer milenio, entre los siglos IX yXI se produciría un abandono de la ocupación del piedemonte producto de cambios climáticos, en tanto que el espacio social se reorganizaría partir del surgimiento de diversos emplazamientos de los grupos en los tramos superiores de las cuencas.

En cuanto al registro cerámico, la mayoría de los antecedentes del área se dedicaron esencialmente a la caracterización tipológica, estableciendo la presencia de fragmentos de los estilos Ciénaga -en sus variedades gris inciso y naranja pintado-, Saujil gris inciso, Aguada -en sus variedades naranja pintado- y Allpatauca (Dlugosz et al. 2009; Mercado 1994; Ortiz Malmierca 2001; Raviña y Callegari 1992), los cuales son coherentes con diversos estudios sobre el periodo en la región, incluyendo los valles de Abaucán, Hualfín, Catamarca, Ambato y Antinaco-Los Colorados (centro-oeste de Catamarca y norte de La Rioja) (Balesta et al. 2015; Callegari et al. 2015; De la Fuente y Vera 2015; Feely et al. 2016; González 1998; Kush 1994; Laguens et al. 2007; Nazar y De la Fuente 2016; Pérez y Heredia 1990; Sempé y Baldini 2011; entre otros). Por otro lado, trabajos recientes (Cahiza 2015; Cahiza et. al. 2017; Sabatini y Garate 2017) en Anillaco, Anjullón y Los Molinos se enfocaron en el estudio de diseños decorativos en las vasijas, y en la disposición de éstas en el espacio. Desde el punto de vista cronológico, también se asocian los diferentes estilos a distintos momentos de ocupación (Cahiza et al. 2017), estableciendo que los inicios del primer milenio se vincularían a la alfarería Gris Incisa/ Excisa (correspondiente con los estilos Cienaga y/o Saujil), y luego, hacia la mitad y fines del primer milenio se presentarían otros componentes cerámicos como Ciénaga Naranja, Aguada y Allpatauca. Algo similar se plantea en registros regionales (Callegari et al. 2013; Ratto et al. 2015), sin embargo, hasta el momento en Uchuquita no se han podido distinguir estratigráficamente estos conjuntos, ya que se presentan mezclados en todas las capas, impidiendo una buena visión diacrónica de los sitios.

Si bien estos antecedentes son importantes, la problemática esencial acerca de la tecnología alfarera en Uchuquita (y zonas cercanas) radica en dos aspectos íntimamente relacionados: la definición de los conjuntos cerámicos, esto es, el conocimiento composicional y tecnológico de los estilos, las fuentes de materias primas, la funcionalidad de vasijas, y el mantenimiento y/o quiebre de "recetas técnicas" por parte de los alfareros durante el periodo. Y por otro lado, el contexto de producción, incluyendo la escala de manufactura y localización de espacios productivos, la circulación de ceramios y sujetos dentro del área (el faldeo oriental de la Sierra de Velasco) y entre ésta y la región. Este panorama complejo requiere de un enfoque de investigación amplio para su comprensión, siendo este trabajo un aporte inicial en el que se plantean diversos interrogantes: ¿Cómo se conforman y cuáles son las características de pastas de los conjuntos cerámicos de los sitios?, ¿existen variabilidades tecnológicas entre ellos?, ¿cuáles y en base a qué factores se decidieron las elecciones técnicas durante las primeras fases de la cadena operativa?, ¿se utilizaron materias primas regionales para la elaboración de cerámicas?, ¿hay presencia de bienes alóctonos?, ¿cuál es la relación de la alfarería con los modos de vida de las comunidades aldeanas de Uchuquita? para resolver estas interrogantes la cadena operativa conforma una herramienta teóricametodológica que permite un análisis meticuloso de las producciones cerámicas, y a partir de los estudios submacroscópicos y microscópicos de pastas es posible un conocimiento detallado acerca de las primeras etapas de manufactura, adentrándose en las tradiciones técnicas que caracterizan a los ceramistas de una sociedad en particular.

\section{Ubicación, Ambiente y Geología}

La cuenca de Anillaco ( $\left.28^{\circ} 50^{\prime} \mathrm{S} / 66^{\circ} 56^{\prime} \mathrm{W}\right)$ se ubica en el sector noreste de la Sierra de Velasco. El paisaje del área presenta un perfil de altos contrastes de relieve, tales como sectores montañosos con un máximo de $4000 \mathrm{msm}$, piedemontes surcados por arroyos (1600/1000 msm) y finalmente una depresión en el fondo de valle desértico (1000 a $800 \mathrm{msm}$ ) limitada por estribaciones del cordón oriental del Velasco -Sierra de La Punta- (Figura 1A). La región posee precipitaciones de $200 \mathrm{~mm}$ anuales y pertenece a la provincia fitogeográfica del monte; sin embargo, por su composición florística, las quebradas se asemejan más al chaco serrano, con un estrato arbóreo dominado por Prosopis sp., Celtis sp., Acacia visco y Lithraea molloides, arbustivo como Acacia sp. y Schinus sp., un estrato herbáceo con gramíneas y cactáceas (Trichocereus sp., Opuntia sp.). En tanto las zonas altas de la sierra presentan sectores de pastizales atractivos para camélidos y ganado moderno (Cabrera 1976).

Geológicamente Anillaco posee una compleja historia desarrollada entre el Ordovícico y Carbonífero, culminando con la orogenia Andina que establece la 


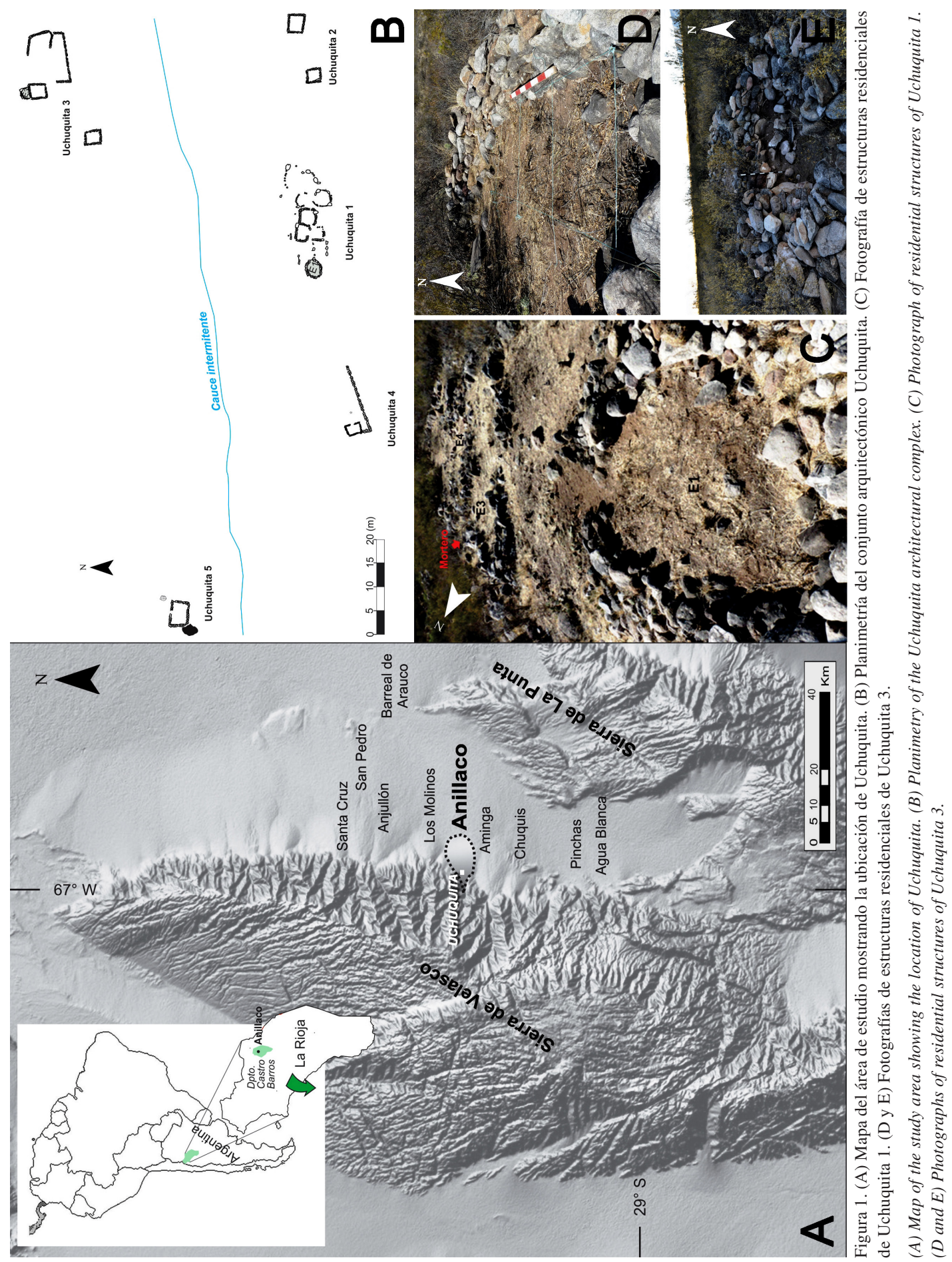


configuración actual de la Sierra de Velasco (Figura 2) (Alasino et al. 2006; Bodenbender 1911; Toselli et al. 2005; entre otros). Forma parte de la morfoestructura de las Sierras Pampeanas, constituida por un basamento cristalino conformado por rocas metamórficas y diversas rocas ígneas. En el sector oriental de la sierra aflora la Formación La Cébila, de edad ordovícica inferior, y se compone fundamentalmente de esquistos y filitas. La zona centro y norte del Velasco se destaca por sus importantes afloramientos de granitos deformados del Ordovícico (Complejo Antinaco), y granitos no deformados de edad carbonífera inferior, entre los que se reconocen los granitos porfíricos Asha, San Blas, Huaco, Sanagasta y La Chinchilla. Anillaco se encuentra en las cercanías del Complejo Antinaco y del Plutón La Costa, ambos al oeste. El Complejo Antinaco se compone de orto-gneises y granitos gneisicos porfiroides, con foliación milonítica que gradan a gneises miloníticos y protomilonitas. Son rocas de composición monzogranítica, granodiorítica y tonalítica, de grano grueso a porfíricas. Su mineralogía se destaca por minerales micáceos, granate, porfiroclástos de cuarzo, feldespatos y microclino. Por otro lado, el Plutón La Costa está constituido por granitos porfíricos a equigranulares de grano grueso. $\mathrm{Su}$ mineralogía está representada por cuarzo, microclino y plagioclasa, junto con biotita, muscovita, turmalina, granate, apatita y circón. En la zona del piedemonte se localizan conos de deyección formados por la acción de los ríos (los Molinos, Anillaco y Aminga) que nacen en la sierra, donde se encuentran depósitos poco consolidados, granulares gruesos, pobremente seleccionados, formados esencialmente en geoformas fluviales y coluviales (González et al. 2008).

\section{Materiales y Metodología}

El conjunto aldeano Uchuquita está integrado por cinco núcleos residenciales de tipo doméstico (Uchuquita 1,2,3,4y 5), compuestos por patios y recintos habitacionales de muro de piedra de planta semicircular y semirectangular (Figura 1B, C y D) (Sabatini y Garate 2017). La alfarería procede de los trabajos de excavación de las unidades domésticas Uchuquita 1 y 3. Ambos pertenecen al componente temporal inicial de las comunidades aldeanas en Anillaco. Un elemento óseo de camélido correspondiente al piso de ocupación de Uchuquita 1 fue datado en $1510 \pm 22$ años AP Cal 542 a 589 DC (D-AMS 028048), en tanto una muestra de carbón vegetal de los contextos de ocupación de Uchuquita 3 ha sido fechado en $1760 \pm 50$ años AP Cal 220 DC a 376 DC (LP-3259)' (Sabatini y Garate 2017; Sabatini y Salminci 2016).

Para el estudio de las pastas del registro se ha utilizado una metodología integral, generando datos que permitan analizar la relación entre lo que el/la ceramista fabricó, el modo en que lo hizo, y las materias primas que utilizó. La unificación de la información morfológica/decorativa, tecnológica y de composición ofrece una aproximación sólida para estudiar las prácticas de producción de la alfarería consumida en el sitio, y reconocer las estrategias de elaboración particulares y compartidas por distintos artesanos (Druc 2013; Puente 2012; Sillar y Tite 2000; entre otros).

La muestra fragmentaria está compuesta por un total de 2.383 tiestos, los cuales fueron agrupados en 54 Unidades de Análisis (UA) o familias cerámicas de acuerdo a aspectos macroscópicos que exhiben los atributos (presencia o no de decoración, tonalidad, características generales de pastas y de tratamientos de superficies) (Orton et al. 1997). Uchuquita 1 posee 36 UA, mientras que Uchuquita 3, 18 UA. Cada UA fue clasificada según recipiente de tipo no restringido (platos y cuencos) y restringidos (ollas), y "conjunto tecnotipológico" decorado (D) y no decorado (ND), asociado a los estilos Aguada, Ciénaga y/o Saujil y Allpatauca reconocidos, así como a otros no asignados hasta el momento (Tabla 1) (Figuras 3 y 4).

Para el análisis con lupa binocular se hizo un muestreo dirigido asistemático y aleatorio (Shennan 1992), seleccionando el $10 \%$ de fragmentos en cada UA, focalizándose en las partes diagnósticas más representativas de cada forma (cuellos, bordes y bases), aunque también en tiestos correspondientes al cuerpo del recipiente. En total se analizaron 477 fragmentos (20\% del registro global) con lupa binocular (Leica Zoom 2000) a partir de corte fresco. Se caracterizó por un lado la matriz: color a ojo desnudo y según Cartilla de Color de Suelos Munsell (1994), textura, fractura y dureza, y cambios de coloración; por otro lado los antiplásticos: identificación -en los casos posibles-, color, tamaño, distribución, densidad y forma o grado de esfericidad, y orientación. Asimismo se describieron las cavidades teniendo en cuenta: distribución, densidad, forma, orientación y tamaño (Cremonte y Bugliani 2006-2009; Rye 1981; Urteaga y Amundaray 2003). A partir de este estudio se conformaron siete estándares o patrones tecnológicos de las pastas cerámicas, los cuales fueron asociados a los distintos estilos y formas reconocidas (Cremonte 1990-1991).

Posteriormente, se realizó un estudio petrográfico de 43 láminas delgadas cerámicas. Cada una de ellas pertenece a una UA, cubriendo prácticamente la totalidad del registro, dejando de lado exclusivamente y arbitrariamente once UA, debido a que se hallaban compuestas por escasos y pequeños tiestos, y pertenecían a estándares de pasta ya muestreados. En ese sentido, la selección se basó en la representatividad de UA de cada estilo tecnológico y formas del universo cerámico, 


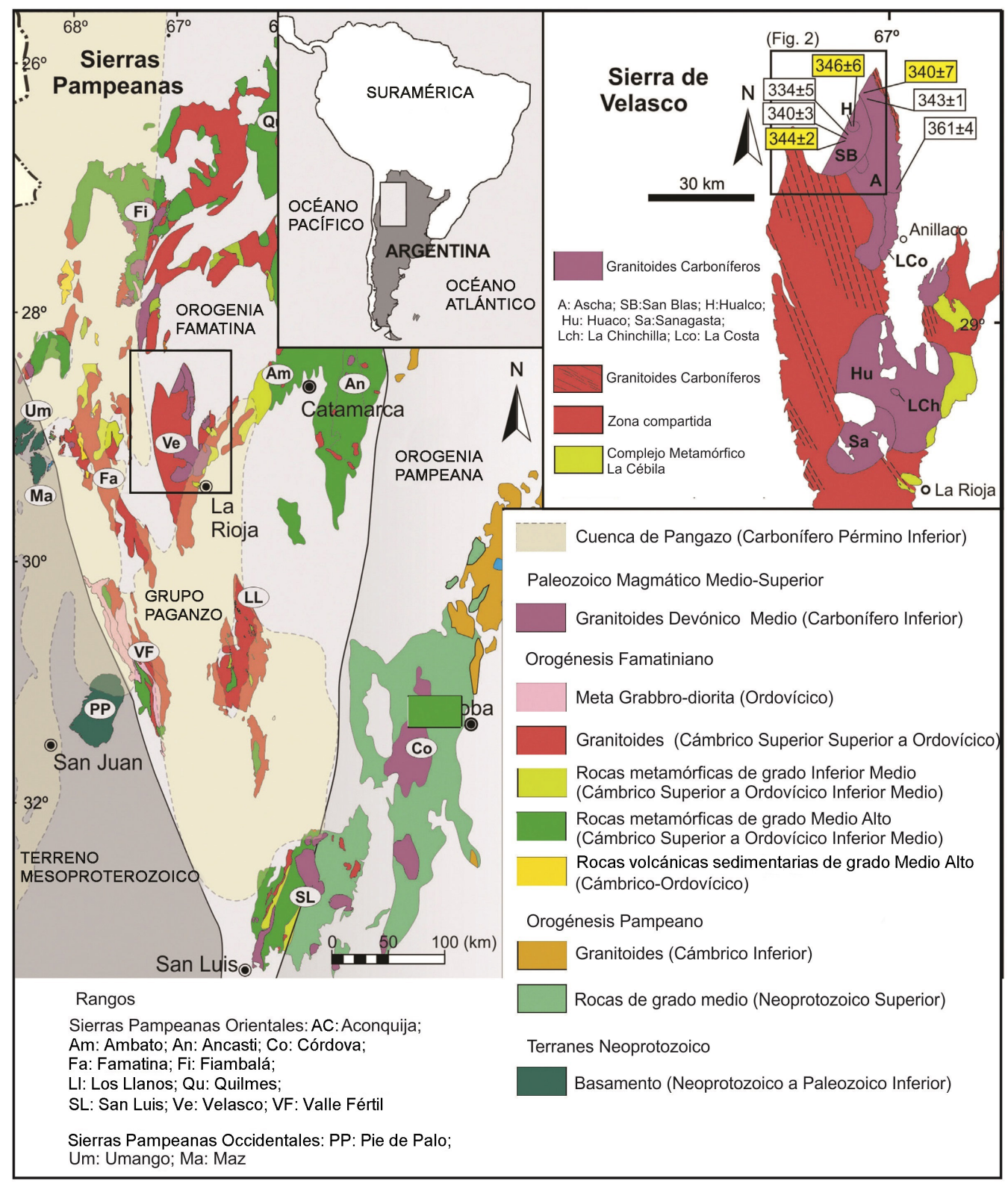

Figura 2. Mapa geológico del área (tomado y modificado de Alasino et al. 2017).

Geological map of the area (taken and modified from Alasino et al. 2017). 
Tabla 1. Muestra cerámica de Uchuquita. Ceramic sample of Uchuquita.

\begin{tabular}{|c|c|c|c|c|}
\hline \multicolumn{2}{|c|}{ Sitio } & Uchuquita 1 & Uchuquita 3 & Total \\
\hline \multicolumn{2}{|c|}{ Fragmentos } & 2125 & 258 & 2.383 \\
\hline \multicolumn{2}{|c|}{ UA } & 36 & 18 & 54 \\
\hline \multirow{4}{*}{ Morfología } & Cuencos & 26 & 9 & 35 \\
\hline & Platos & 1 & 0 & 1 \\
\hline & Ollas & 9 & 5 & 14 \\
\hline & Indeterminado & 0 & 4 & 4 \\
\hline \multirow{2}{*}{ Conjunto tecnotipológico } & Decorado & 25 & 14 & 39 \\
\hline & No decorado & 11 & 4 & 15 \\
\hline \multirow{5}{*}{ Estilo } & Aguada & 1 & 3 & 4 \\
\hline & Ciénaga/Saujil Gris & 17 & 8 & 25 \\
\hline & Ciénaga Naranja & 9 & 3 & 12 \\
\hline & Allpatauca & 6 & 4 & 10 \\
\hline & Indeterminado & 3 & 0 & 3 \\
\hline
\end{tabular}

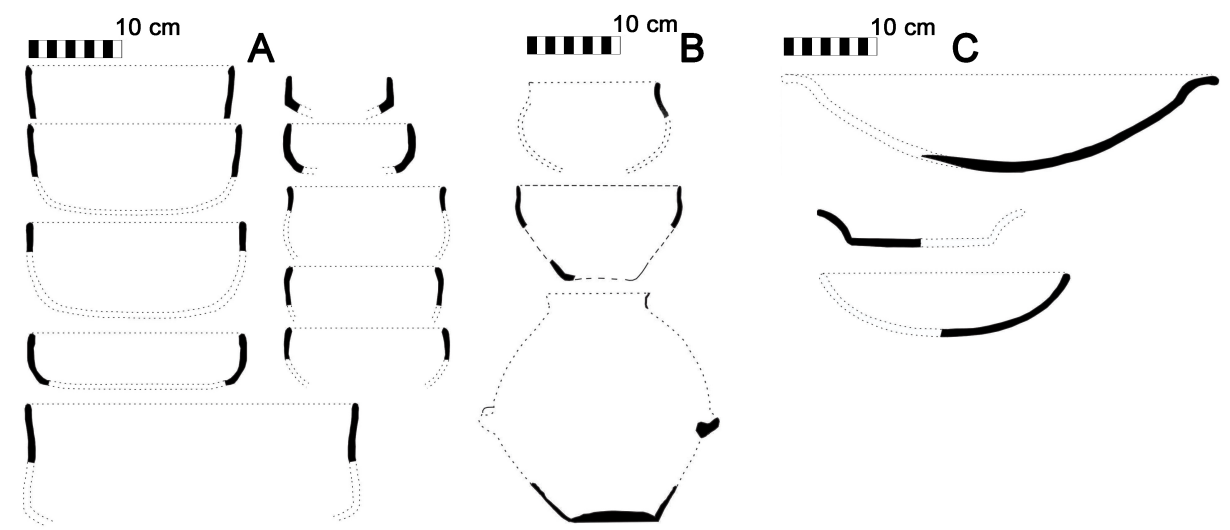

Figura 3. Formas cerámicas representativas del registro de Uchuquita 1 y 3. (A) Cuencos o pucos. (B) Ollas. (C) Platos.

Representative ceramic forms from Uchuquita 1 and 3.(A) Bowls or pucos. (B) Pots. (C) Dishes.

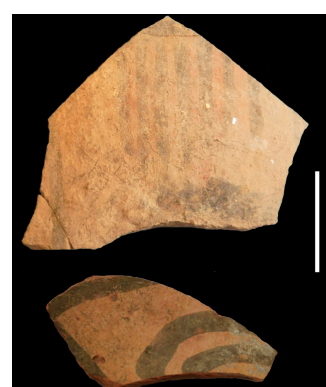

A

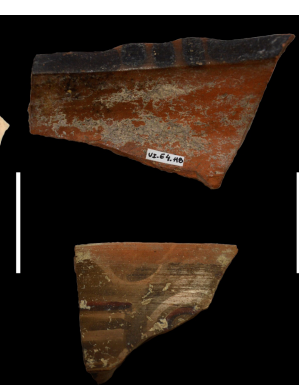

B

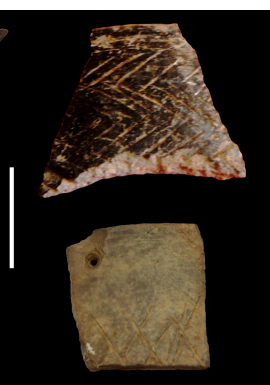

C

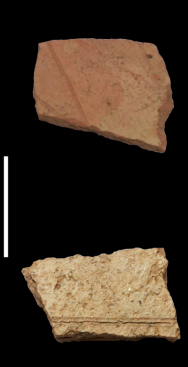

D

\section{$10 \mathrm{~cm}$}

Figura 4. Estilos cerámicos de Uchuquita. (A) Ciénaga Naranja. (B) Aguada. (C) Ciénaga y/o Saujil Gris. (D) Allpatauca.

Uchuquita ceramic styles. (A) Ciénaga Naranja. (B) Aguada. (C) Ciénaga y/o Saujil Gris. (D) Allpatauca. 
siendo la mayor parte tiestos correspondientes a cuerpos y bordes de vasijas.

Para el análisis se utilizó microscopio LEICA MPS 522, orientando la observación hacia: la identificación y cuantificación (300 puntos por lámina delgada) de las distintas especies minerales, fragmentos líticos, y otros antiplásticos; la determinación de proporción de matriz, cavidades e inclusiones no plásticas a partir de estimaciones de área mediante cartilla de cuantificación por comparación visual; la descripción de textura de fondo de pasta; el reconocimiento de la orientación de los constituyentes y cavidades; la caracterización del tamaño, redondez, esfericidad, selección y madurez textural y mineralógica de materiales clásticos; la detección de minerales secundarios; y la caracterización de cavidades (Cuomo di Caprio y Vaughan 1993; Curtois 1976; González de Bonaveri et al. 2000; Livingstone Smith 2007; Middleton et al. 1985; Raith et al. 2012; Stoltman 1989). Esto permitió definir los estándares de pastas, logrando de manera no automatizada un mayor ajuste de las agrupaciones, además de la generación un modelo de clasificación con potencial flexibilidad ante el incremento de muestras y datos producto del avance de investigaciones (Middleton et al. 1991). Sin embargo, para entender mejor la relación entre las pastas, su uniformidad o variabilidad, se practicó un examen estadístico descriptivo y multivariado de los datos composicionales obtenidos. Para ello se realizó un análisis de clúster empleando el agrupamiento centroide y distancia euclidiana al cuadrado (o método Ward), utilizando el programa Past 3.02 (Baxter y Jackson 2001; Shennan 1992). El procedimiento presenta algunas dificultades y limitaciones derivadas del lenguaje matemático aplicado, y se relacionan con la problemática de separación y validez de los grupos generados, por lo que hacen que el análisis de conglomerados sea un procedimiento básicamente exploratorio, y que requiera la contrastación de los resultados a partir de la observación del material alfarero con diferentes métodos ópticos, y otros estudios arqueométricos (Albero Santacreu 2014).

\section{Resultados}

\section{Análisis submacroscópico de pastas}

La observación submacroscópica de pastas permitió la distinción de siete estándares (E) o patrones en el registro global, siendo los más abundantes el 2, 3 y 4 (Tabla 2). A nivel de sitio, en Uchuquita 1 se destaca la presencia de los estándares 2, 6 y 7, mientras que en Uchuquita 3 existe mayor representatividad del estándar 5 frente al resto, los cuales se encuentran en proporciones semejantes, además de que no se hallan cerámicas con los estándares 6 y 7 (Figura 5). En su vínculo con los estilos, no se observa una distinción de estándares de pasta para piezas decoradas (D) y no decoradas (ND), aunque si se advierte mayor diversidad de estándares dentro del estilo Cíénaga y/o Saujil Gris (estándares 4, 5 y 6). En ese sentido, el conjunto de estos estándares es el mayor representado tanto para Uchiquita 1 como Uchuquita 3, alcanzando el $42 \%$ del registro global. En cuanto a la relación de las pasta con las formas, se reconocen los mismos estándares para la confección de las diferentes morfologías de piezas.

El registro alfarero posee pastas de tonalidad naranja rojiza (10YR 6/8), marrón rojiza (5YR 5/4), gris (10YR 5/1) y grisácea marrón (10YR 6/2) (Munsell Soil Color Charts 1994). Casi la totalidad del conjunto no exhibe cambio de coloración en toda la secuencia cromática desde las superficies hasta el núcleo de pasta, exhibiéndose a nivel global una gran regularidad cromática. Los estándares en los que se ha reconocido variabilidad en la coloración son el 5, con el desarrollo de un núcleo grisáceo o más oscuro con márgenes rectos; y los estándares 2, 3 y 4, en donde se advierte en escasos tiestos transformación cromática hacia tonos más oscuros entre el núcleo y la superficie interna o externa de la pieza. Las texturas de pastas son fundamentalmente compacta y compacta/porosa, y presentan una categoría de dureza dura y muy dura, y una fractura laminar, cortante e irregular (Figura 6).

En cuanto a la composición, en la mayoría de los estándares se reconocen constituyentes comunes como cuarzos (qtz), feldespatos (feld.), micas y diversos litoclastos (lit.). En los estándares 1, 2 y 7 se identifican rocas azules y grises; el 3 se reconoce por la presencia de grandes cristales de mica, además de cuarzo, feldespatos y fragmentos de roca grises; los estándares 4, 5 y 6 se exhiben por la gran carga de minerales félsicos y líticos blancos, negros y grises. En general los antiplásticos poseen formas esféricas y prismáticas, sub redondeadas y sub angulosas. Las diferencias mayores radican en el tamaño, la distribución y la densidad. La granulometría de los cristales comúnmente es de muy fina a muy gruesa $(0,05-2 \mathrm{~mm})$, aunque mayoritariamente fina $(0,2 \mathrm{~mm})$. El estándar con tamaño de grano mayor es el $3(0,5 \mathrm{~mm}$ promedio), mientras que aquellos con tamaño menor son el 5 y $6(0,05 \mathrm{~mm}$ promedio) y 1 ( $0,07 \mathrm{~mm}$ promedio). En cuanto a la distribución y la densidad, normalmente es pobre, y del $5 \%$ y $10 \%$. En los estándares 1 y 2 es muy pobre y entre $5 \%$ y $20 \%$, en el estándar 3 y 4 pobre y del $20 \%$, y en los restantes $-5,6$ y 7 - buena y entre $5 \%$ y $10 \%$ de densidad.

Por su parte, las pastas presentan predominantemente cavidades de forma redonda, aunque también alargada, además de moderada densidad (alrededor del 10\%), distribución equilibrada, y un tamaño que no supera los $0,4 \mathrm{~mm}$. El estándar con mayores cavidades es el 3, mientras que los que muestran menor densidad son el 1 y 6. 


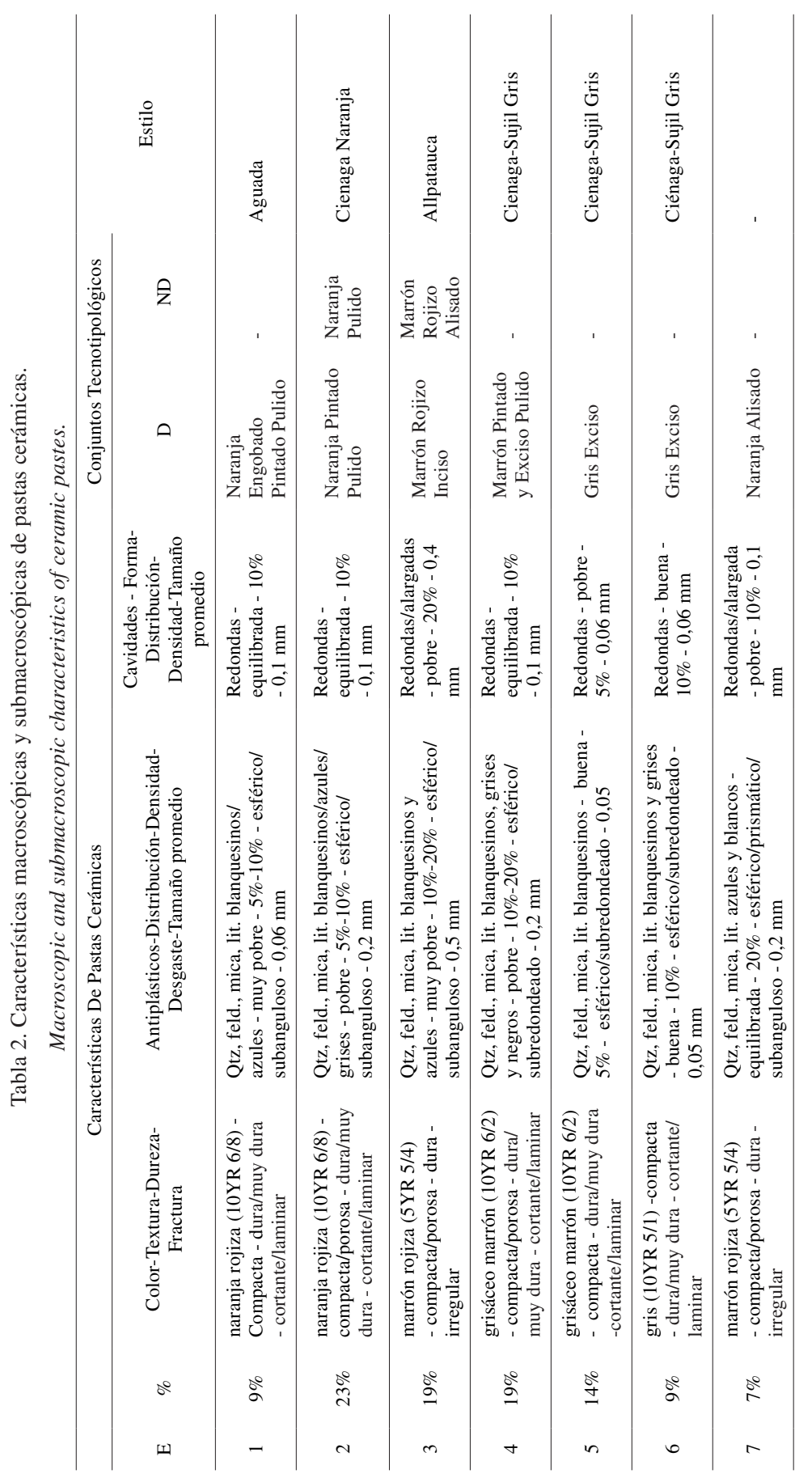




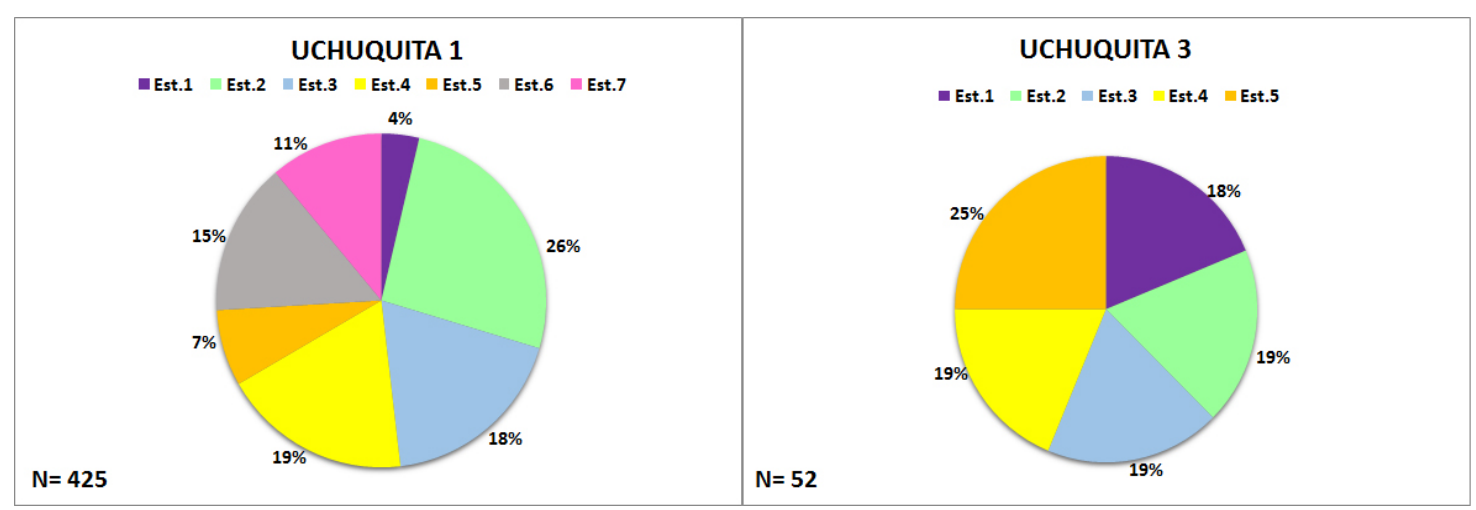

Figura 5. Distribución porcentual de estándares de pasta en Uchuquita 1 y 3. N= número de fragmentos observados en lupa binocular.

Percentage distribution of paste fabrics in Uchuquita 1 and 3. N= number of fragments observed with binocular loupe.
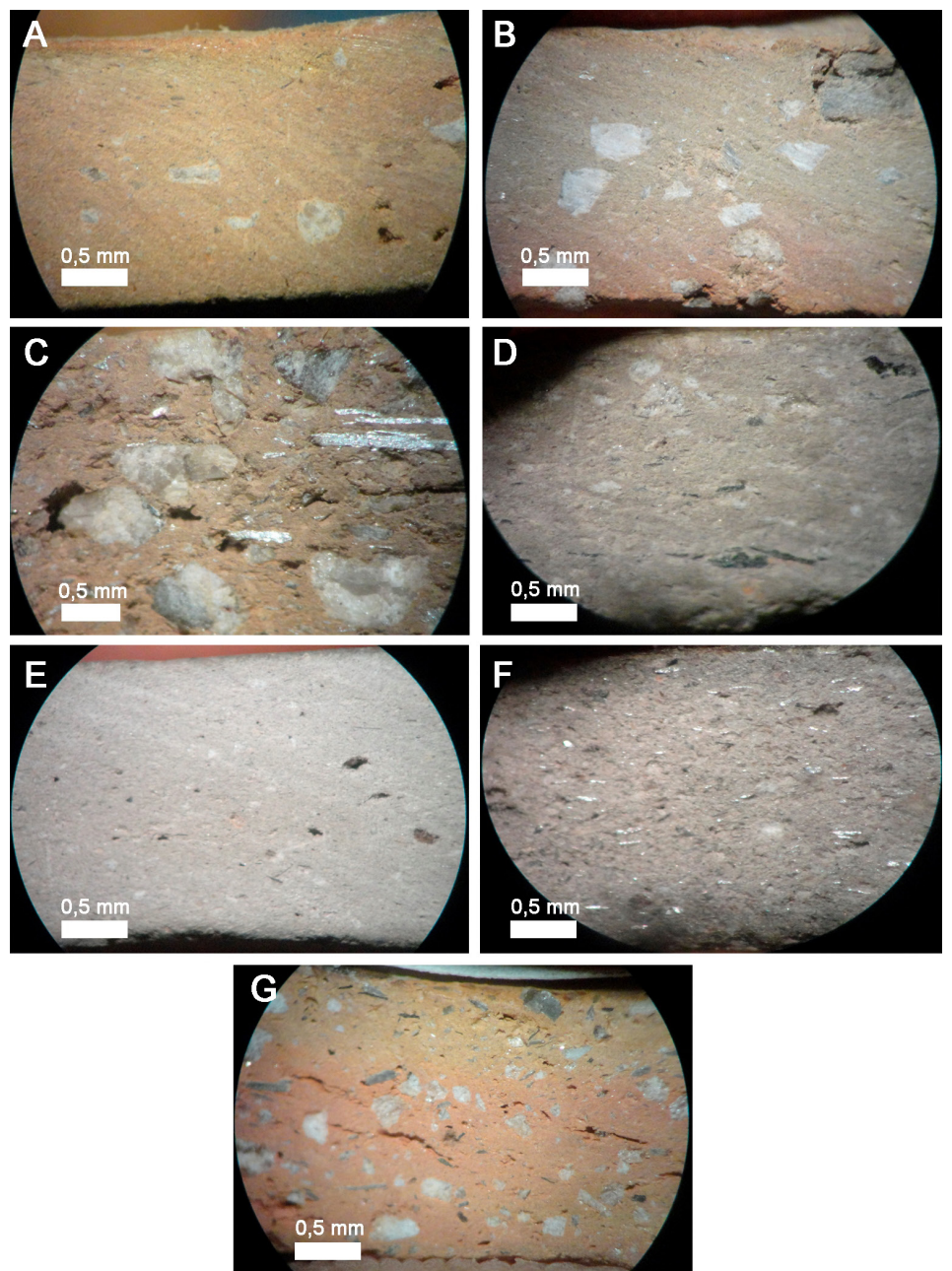

Figura 6. Fotografías con lupa binocular de los estándares cerámicos del registro. Est.= estándar. Photographs with binocular loupe of Uchuquita ceramic fabrics. Est. $=$ fabric. 


\section{Análisis microscópicos de pastas}

Los resultados sobre el análisis petrográfico de las 43 secciones delgadas establecen la presencia de pastas composicionalmente homogéneas, con escasa variación (Tabla 3). En prácticamente la totalidad de la muestra se identifican estructuras de fondos de pasta microgranosas/criptofilitosas, con abundantes microcristales de minerales félsicos y máficos, entre ellos cuarzos, feldespatos, biotita, muscovita, minerales opacos, óxidos, piroxenos y anfíboles. Dado el tamaño menor a $0,05 \mathrm{~mm}$ (limo grueso) de estos antiplásticos es altamente dificultosa su identificación, así como el reconocimiento de sus formas, las cuales parecerían ser anhedrales y subhedrales, de hábito prismático, fibroso y planar.

Los antiplásticos de mayor tamaño -a partir de los 0,06 mm- son minerales félsicos como cuarzo monocristalino (Qtz) (21,7\%) -en algunos casos con fracturas planares, lamelas de deformación y bandas de plegamiento producto de metamorfismo-, microclino (Mi) $(1,23 \%)$, plagioclasa $(\mathrm{Pl})(8,65 \%)$ y feldespatos potásicos (Fk) $(8,89 \%)$-varios de ellos alterados a sericita, macla polisintética y zonación óptica-, con forma euhedral y subhedral, de hábito prismático y planar. También se distingue importante cantidad de biotita (Bt) $(11,57 \%)$ y muscovita (Ms) $(16,1 \%)$, ehuedrales y de hábito fibroso, además de óxidos de hierro (Ox) (5,24\%), minerales opacos $(7,27 \%)$, anfíboles (Anf) $(0,55 \%)$ y piroxenos (Px) $(0,68 \%)$, con formas subhedrales y hábito prismático. Este conjunto de minerales representan el $81,88 \%$ del registro de antiplásticos que exhiben las pastas. Por otro lado, las cerámicas tienen importante carga de fragmentos de rocas plutónicas $(\mathrm{Lp})(8,12 \%)$-granitos y granodioritas porfídicos-, muchos de los cuales se hallan con bajo y moderado grado de metamorfismo ( $\mathrm{Lm})(2,63 \%)$. Estos litoclastos suman a nivel global el 10,75\% de los antiplásticos, y se les asocian en muy baja proporción otros constituyentes petrológicamente relacionados como vulcanitas félsicas (Lvf) $(1,97 \%)$-vitroclastos alterados a cuarzo y feldespato-, vidrio volcánico (Vv) $(0,97 \%)$, y escasas rocas volcánicas máficos (Lvm) $(0,19 \%)$. Asimismo, se reconocen en baja proporción nódulos arcillosos (Nar) (4,16\%). Todo este grupo de rocas y productos accesorios, que en conjunto representan el 18,12\% del total de antiplásticos, posee esfericidad esférica/subdiscoidal y redondez subangulosa/subredondeada, tal como se advierte a observación submacroscópica.

Estas características permiten advertir una selección clástica moderada y pobre de los antiplásticos, denotando una madurez textural inmadura de los sedimentos utilizados para las pastas. La distribución de desgrasantes en la matriz se exhibe esencialmente equilibrada y pobre, y con una orientación aleatoria. En cuanto al tamaño, a escala microscópica se observa que algunos cuarzos poseen un tamaño máximo de $1,3 \mathrm{~mm}$ (arena muy gruesa), aunque el promedio es de 0,125 $\mathrm{mm}$ (arena fina), mientras que los granitos y granitoides alcanzan los $2 \mathrm{~mm}$ (gránulo), promediando en $0,2 \mathrm{~mm}$ (arena media) (Figura 7). Las cavidades se identifican con formas mayormente redondas (88\%) y escasamente alargadas (12\%), con distribución equilibrada (93\%) y pobre (7\%), generalmente sin conexión, con orientación aleatoria y con un tamaño promedio de $0,1 \mathrm{~mm}$. El único estándar que se diferencia de estas características es el estándar 3, donde las cavidades se advierten alargadas y conectadas, llegando a ostentar un tamaño máximo de $0,8 \mathrm{~mm}$.

Respecto a los estándares de pastas (E), a nivel microscópico se advierte, en primer lugar, una cierta similitud en la composición general de los estándares 1 y 2 . En ambos casos se reconoce gran cantidad de biotita, muscovita, minerales opacos, óxidos, cuarzo, feldespato potásico, plagioclasa y rocas plutónicas. La diferencia entre estos estándares está signada principalmente por las estructuras de fondo de matriz, microgranosa/criptofilitosa en el primer caso, y microgranosa/pseudolepidoblástica en el estándar 2. Asimismo, se advierte mayor presencia de litoclastos graníticos metamórficos en el estándar 1 -constituyente que también lo discrimina del registro global-, un tamaño máximo de antiplástico de $0,07 \mathrm{~mm}$ (arena muy fina) y una densidad no supera el $10 \%$; mientras que el estándar 2 se caracteriza por exhibir alta cantidad de muscovita y granitos, así como un tamaño promedio de antiplástico mayor a $0,1 \mathrm{~mm}$, y una densidad entre $10 \%$ y $20 \%$. El estándar 3 se asemeja a los anteriores desde la composición, aunque se reconoce claramente por poseer el tamaño mayor de antiplásticos en el registro, alcanzando algunos cuarzos y rocas los $2 \mathrm{~mm}$, distribuidos de manera pobre. También es casielúnicoen presentar biotita y muscovita con tamaños que superan el milímetro, y mostrar las cavidades más grandes del registro de pastas, con forma alargada y unidas entre sí a lo largo de la matriz. En estos tres estándares se advierten pastas de tonalidad naranja/rojiza y marró/ rojiza, dado probablemente por un aporte importante de óxido de hierro y una cocción oxidante (Figura 8). El estándar 4, si bien se equipara a los estándares 1 y 2 por la moderada/baja densidad de antiplástico, se destaca y diferencia por presentar matriz marrón con mucha birrefringencia, propia de una estructura de fondo pseudolepidoblástica, probablemente dada por abundantes microcristales de muscovita y óxidos, y quizás la cocción. Además, exhibe abundantes plagioclasas y feldespatos potásicos, y tiene un tamaño promedio de antiplástico de $0,1 \mathrm{~mm}$. Los estándares 5 y 6, también pertenecientes al estilo Ciénaga/Saujil Gris, 
Tabla 3. Análisis modal de pastas cerámicas estudiadas por petrografía. Los valores se expresan en porcentajes.

Modal analysis of ceramic pastes studied by petrography. The values are expressed in percentages.

\begin{tabular}{|c|c|c|c|c|c|c|c|c|c|c|c|c|c|c|c|c|c|c|}
\hline $\mathrm{N}^{\circ}$ & $\begin{array}{l}\text { Fragmento } \\
\text { Cerámico }\end{array}$ & $\mathrm{E}$ & Qtz & $\mathrm{Pl}$ & $\mathrm{Mi}$ & $\mathrm{Fk}$ & Ms & $\mathrm{Bt}$ & Anf & Px & Mo & Ox & Lvf & Lvm & $\mathrm{Vv}$ & $\mathrm{Lp}$ & $\mathrm{Lm}$ & Nar \\
\hline 1 & A 1.33 & 2 & 16,16 & 7,16 & 0 & 14 & 17,33 & 10 & 3,66 & 2,33 & 5,33 & 4,83 & 0 & 0 & 1,66 & 10,8 & 0,33 & 6,33 \\
\hline 2 & A1.35 & 2 & 20,16 & 12,33 & 0 & 8 & 18,66 & 8,16 & 0,66 & 0,33 & 7,16 & 5,33 & 1 & 0 & 1,33 & 12,7 & 0 & 4,16 \\
\hline 3 & $\mathrm{~A} 2.7$ & 2 & 17,33 & 9,66 & 0 & 15,66 & 21,33 & 10,8 & 0 & 2 & 5,33 & 3,83 & 0 & 0 & 0 & 9,33 & 1 & 3,66 \\
\hline 4 & A2.41 & 2 & 18,16 & 8,33 & 5 & 14,33 & 13,16 & 18,3 & 0 & 0 & 7,16 & 2,16 & 0 & 0 & 1,16 & 8,66 & 0,66 & 2,83 \\
\hline 5 & A2.61 & 3 & 22,16 & 3,33 & 0 & 7,66 & 16,66 & 18,7 & 0,16 & 0 & 6,33 & 8,33 & 0 & 0 & 0 & 10 & 1,33 & 5,33 \\
\hline 6 & A2.79 & 7 & 19,16 & 13,16 & 0 & 7,16 & 16,83 & 9,66 & 1,16 & 0,66 & 7,83 & 4 & 4,16 & 0 & 1,33 & 11 & 0 & 3,83 \\
\hline 7 & A 3.1 & 3 & 20,16 & 11 & 2,33 & 7,66 & 19,33 & 12,2 & 0 & 0 & 3,66 & 5,16 & 0 & 0 & 0 & 13,2 & 1,66 & 3,66 \\
\hline 8 & A 3.3 & 1 & 17,16 & 8,33 & 0,16 & 15,66 & 16,16 & 9,66 & 4,16 & 3,33 & 6 & 3,16 & 0 & 0 & 1,16 & 8,33 & 1,33 & 5,33 \\
\hline 9 & A 3.35 & 6 & 25 & 9,33 & 0 & 12,33 & 9,66 & 10,7 & 0 & 2 & 9,16 & 4,33 & 0,83 & 0 & 0 & 8,33 & 1,16 & 7,16 \\
\hline 10 & A3.46 & 4 & 26,33 & 14,16 & 0 & 14,33 & 12,83 & 7,66 & 0 & 1 & 8,66 & 2,33 & 0 & 0 & 0 & 1,66 & 7,33 & 3,66 \\
\hline 11 & A 3.77 & 4 & 25,16 & 13,83 & 0,16 & 15,66 & 11,33 & 8,33 & 0 & 0,33 & 7,16 & 4,66 & 0 & 0 & 0 & 2,83 & 5,66 & 4,83 \\
\hline 12 & A3.91 & 7 & 19,66 & 8 & 2,16 & 10,83 & 13,83 & 17,3 & 0 & 0,33 & 9,33 & 3,16 & 5,16 & 0 & 1,66 & 6,66 & 0,16 & 1,66 \\
\hline 13 & A4.16 & 4 & 21 & 10,16 & 3 & 8,16 & 18,66 & 10,7 & 0 & 0 & 5,66 & 4,16 & 0 & 0 & 0 & 12 & 1,66 & 4,83 \\
\hline 14 & A 4.45 & 2 & 20,16 & 4,33 & 0 & 10 & 21,16 & 13,2 & 0 & 0 & 7,33 & 6,16 & 3,16 & 0 & 0 & 9,33 & 1 & 4,16 \\
\hline 15 & B1.13 & 4 & 23,33 & 8,83 & 0 & 12,33 & 16,33 & 7,33 & 0,66 & 0,16 & 7,83 & 4,83 & 0,33 & 0 & 1,16 & 7,16 & 3,33 & 6,33 \\
\hline 16 & B1.53 & 6 & 19,83 & 3 & 0 & 11,66 & 21,83 & 14,8 & 0 & 0,33 & 9,66 & 6,16 & 0 & 0 & 0 & 5,66 & 1,66 & 5,33 \\
\hline 17 & B1.86a & 4 & 22,33 & 9,16 & 0 & 13,16 & 16,33 & 11,7 & 1,33 & 1,16 & 8,16 & 4,66 & 1,66 & 1,33 & 2,16 & 3 & 2,16 & 1,66 \\
\hline 18 & B $1.86 \mathrm{~b}$ & 7 & 17,83 & 9,66 & 1,66 & 7,66 & 15,66 & 16,8 & 0,16 & 0,33 & 5,33 & 2,16 & 7,16 & 0 & 1,83 & 4,66 & 2,66 & 6,33 \\
\hline 19 & B2.2 & 5 & 24,66 & 7,83 & 0 & 7,83 & 11,83 & 10,2 & 1 & 3 & 11,83 & 7,33 & 2,16 & 0 & 2 & 5,66 & 0,83 & 3,83 \\
\hline 20 & B2.82 & 2 & 28,16 & 8,66 & 0 & 9,66 & 17,66 & 12,3 & 0,16 & 1 & 7,83 & 3,16 & 5,83 & 0 & 0,33 & 2,66 & 0,16 & 2,33 \\
\hline 21 & B2.84 & 3 & 15,33 & 9,33 & 3,33 & 4,66 & 15,66 & 19,3 & 0,33 & 0 & 6,33 & 6,66 & 0 & 0 & 0 & 4,33 & 7,33 & 7,33 \\
\hline 22 & B3.72 & 3 & 20,83 & 4,33 & 0 & 6,16 & 16,83 & 18,7 & 0,66 & 0,16 & 5,66 & 7,83 & 0 & 0 & 0 & 11,8 & 0,16 & 6,83 \\
\hline 23 & C1.4 & 6 & 20,83 & 10,16 & 0,87 & 5,16 & 14,66 & 13,7 & 0,33 & 0,16 & 8,33 & 3,16 & 5,16 & 0 & 1,66 & 7,83 & 5,66 & 2,33 \\
\hline 24 & C3.8 & 2 & 19,16 & 11,16 & 0 & 7,66 & 16,33 & 7,83 & 1,33 & 0,66 & 6,16 & 6,83 & 1,33 & 0 & 1,83 & 11,8 & 1 & 6,83 \\
\hline 25 & C 3.13 & 6 & 19 & 10,66 & 1,83 & 7,83 & 13,66 & 12,7 & 0,16 & 0,33 & 7,83 & 2,83 & 7,16 & 0 & 0,83 & 6,66 & 4,66 & 3,83 \\
\hline 26 & C 3.21 & 5 & 31,16 & 9,83 & 0 & 10,16 & 16,33 & 11,3 & 0 & 2,66 & 6,16 & 2,33 & 6,33 & 0 & 0,16 & 1,83 & 0,33 & 1,33 \\
\hline 27 & C1.32 & 3 & 20,33 & 6,16 & 0 & 5,66 & 13,16 & 11,8 & 0 & 3,66 & 12,33 & 8,33 & 1,83 & 0 & 1,16 & 9,83 & 1,33 & 4,33 \\
\hline 28 & CON $20-30.2$ & 1 & 17,66 & 10,66 & 6,83 & 11,33 & 17,66 & 5,33 & 0 & 0 & 6,83 & 4,66 & 0 & 0 & 0 & 9 & 7,33 & 2,66 \\
\hline 29 & CON 20-30.3 & 4 & 21,33 & 7,66 & 1,83 & 7,83 & 15,33 & 6,16 & 1,66 & 0 & 8,66 & 7,33 & 2,33 & 0 & 2,66 & 10 & 1,33 & 5,83 \\
\hline 30 & CON 20-30.7 & 3 & 20,33 & 12,16 & 3,66 & 6,33 & 17,33 & 10,8 & 0 & 0 & 3,66 & 5,83 & 0 & 0 & 0 & 15 & 0 & 4,83 \\
\hline 31 & A 1.4 & 4 & 26,33 & 6,66 & 2,16 & 5,83 & 20,33 & 4,16 & 0 & 0 & 10,16 & 1,33 & 2,66 & 0 & 1,33 & 6,66 & 7,16 & 5,16 \\
\hline 32 & A1.12 & 2 & 21,16 & 11,33 & 1 & 7,66 & 16,33 & 14,7 & 3 & 0 & 5,83 & 3,66 & 1 & 0 & 1,16 & 9,16 & 1,33 & 2,66 \\
\hline 33 & A2.17 & 5 & 30,16 & 4,33 & 1,66 & 3,83 & 19,16 & 11,8 & 0,66 & 0,66 & 6,33 & 9,33 & 1,33 & 0,33 & 3,83 & 0,66 & 4,66 & 1,16 \\
\hline 34 & B1.1 & 5 & 27,33 & 4,33 & 0 & 5,66 & 19,33 & 14,8 & 0 & 1 & 5,83 & 9,66 & 1,16 & 2 & 3,66 & 2,16 & 1 & 2 \\
\hline 35 & B 1.2 & 2 & 22,33 & 6,66 & 0 & 6,83 & 17,66 & 7,16 & 0 & 0 & 8,16 & 7,16 & 3,66 & 0 & 1,66 & 11,8 & 1,16 & 5,66 \\
\hline 36 & B 1.7 & 5 & 29,33 & 3 & 0 & 9,66 & 15,66 & 7,83 & 0 & 1,16 & 6,83 & 4,16 & 3,33 & 0 & 0,33 & 10,2 & 4,33 & 4,16 \\
\hline 37 & B1.11 & 5 & 22,16 & 8,66 & 0,66 & 7,33 & 16,16 & 14,3 & 0 & 0 & 5,16 & 9 & 1,16 & 1,83 & 0,66 & 7,16 & 3,66 & 2 \\
\hline 38 & B1.20 & 1 & 16,33 & 6,33 & 3,33 & 7,66 & 10,16 & 17,8 & 0 & 0 & 10,16 & 7,16 & 3,66 & 0 & 0 & 4,83 & 8,83 & 3,66 \\
\hline 39 & B1.21 & 1 & 23,33 & 6,66 & 0 & 4,16 & 17,16 & 10,7 & 0 & 0 & 8,33 & 5,33 & 4,66 & 0 & 2,16 & 7 & 7,33 & 3,16 \\
\hline 40 & B2.9 & 3 & 20,66 & 4,33 & 5 & 6,16 & 13,16 & 10,7 & 2,16 & 0,33 & 7,16 & 7,33 & 0 & 0 & 0,16 & 14,3 & 2,16 & 6,33 \\
\hline 41 & B2.28 & 4 & 21,16 & 12,83 & 2,16 & 10 & 14,33 & 10,2 & 0 & 0 & 6,16 & 4,33 & 1,66 & 0 & 0 & 8,33 & 2,16 & 6,66 \\
\hline 42 & B2.35 & 2 & 23,66 & 10 & 1,16 & 5,33 & 13,16 & 11,3 & 0 & 0 & 10,16 & 4,16 & 3,33 & 3 & 2,16 & 8,66 & 3,16 & 0,66 \\
\hline 43 & B2.49 & 3 & 19,33 & 14,33 & 3,16 & 5,66 & 16,33 & 6,33 & 0,16 & 0 & 3,66 & 7,33 & 1,66 & 0 & 0 & 16,2 & 3,16 & 2,66 \\
\hline
\end{tabular}




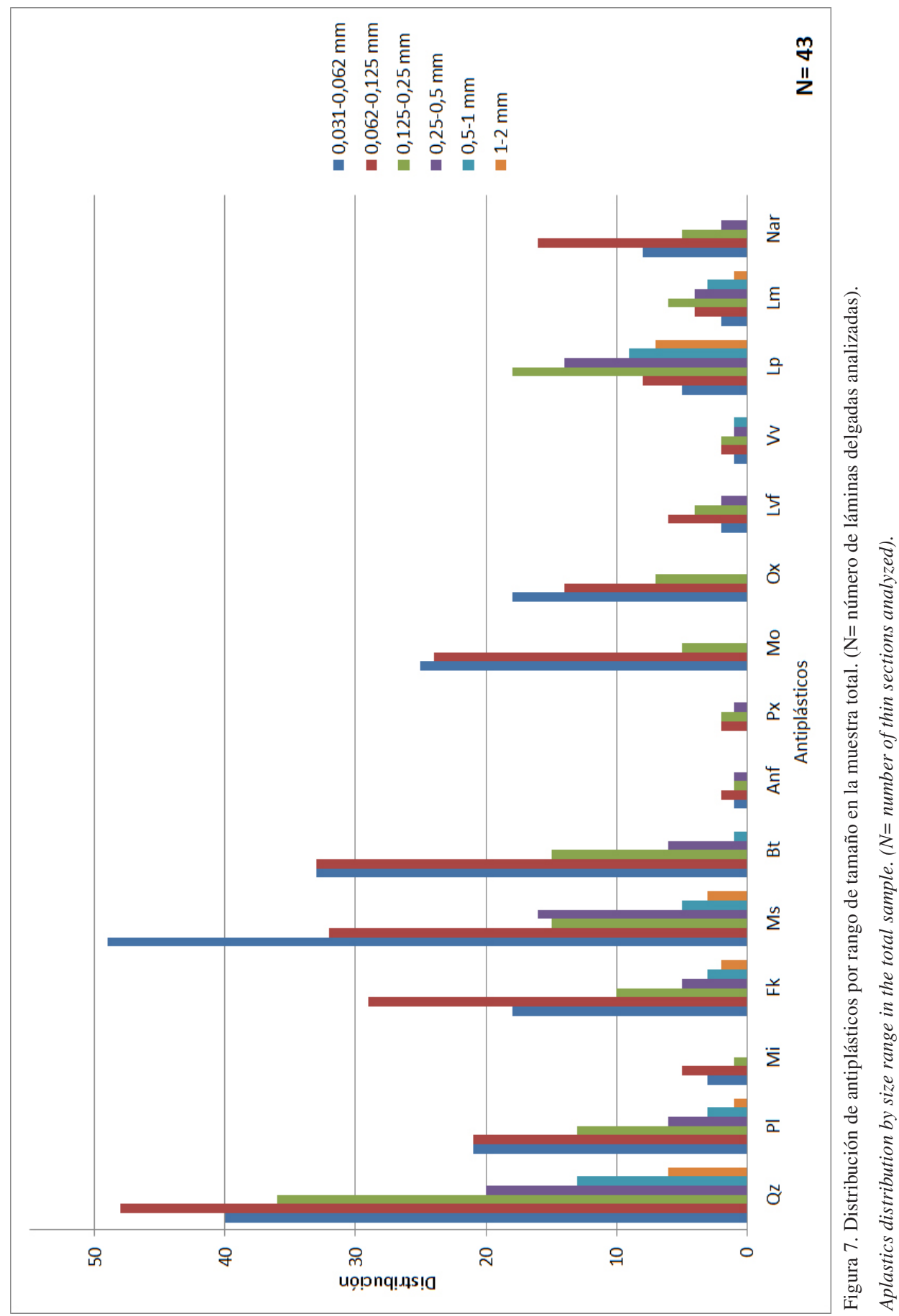


poseen una moderada/baja densidad de antiplásticos, y se asemejan al anterior desde los composicional, una alta presencia de minerales félsicos, principalmente cuarzo (estándar 5), asociado a escasa carga de granitos, granitoides deformados (estándar 6), óxidos, piroxenos y vidrio volcánico. Se diferencian del estándar 4 en que poseen estructuras de fondo de pasta microgranosa/ pseudolepidoblástica, con poca birrefringencia, además de un tamaño promedio de antiplástico levemente menor $(0,06 \mathrm{~mm})$ (Figura 9). Finalmente, el estándar 7 se caracteriza por su matriz rojiza y la abundancia de cuarzo, feldespato potásico, minerales opacos, litoclastos plutónicos, y principalmente vidrio volcánico y rocas volcánicas félsicas (vitroclastos alterados a cuarzo y feldespato), diferenciándose del resto del registro (Figura 10).

La distinción proporcional entre las matrices, inclusiones antiplásticas y cavidades dentro de las pastas es un aspecto difícil de estipular (Cremonte y Bugliani 2006-2009; González de Bonaveri et al. 2000; Stoltman 1989; entre otros), dado que muchas veces no es posible distinguir una relación unimodal o bimodal en las características de los antiplásticos. En este caso la discriminación es compleja además porque, salvo excepciones, el tamaño de los constituyentes es generalmente entre fino y muy fino, y las pastas suelen ostentar los mismos antiplásticos y sus características en minerales menores y mayores a $0,06 \mathrm{~mm}$. Para los estándares 2, 4, 6, 7 y fundamentalmente 3 se observa una relación bimodal de los antiplásticos, sugiriendo además proporciones de 70/20/10 y 80/10/10, es decir cerámicas con abundante matriz, moderada y baja densidad de inclusiones antiplásticas tamaño arena fina, y escasas cavidades. El estándar 3 es el que exhibe mayor carga de inclusiones, con una relación de 60/20/20. Por su parte, los estándares 1 y 5 no advierten claramente la relación bimodal de antiplásticos, sino más bien una homogeneidad general en el tamaño y las características.

El análisis estadístico multivariado exploratorio a partir de la composición petrográfica permitió el agrupamiento de tiestos emparentados de acuerdo al porcentaje de representación y varianzas existentes de las distintas variables analizadas, esencialmente los litoclastos plutónicos y plutónicos metamorfizados y su material accesorio, y los minerales félsicos, máficos, opacos y óxidos (Figura 11). En primer lugar se identifica, por un lado, un Grupo 1, con gran cantidad de muestras $(\mathrm{N}=18)$ contenidas en los subgrupos A y B, ambos caracterizados por presentar los tiestos con la mayor carga de granitos y granitoides, y líticos volcánicos félsicos. Por otro lado, el Grupo 2 se compone de dos subgrupos (C y D), diferenciados por contener la mayor cantidad de minerales félsicos, principalmente feldespato potásico. Los grupos 1 y
2 poseen el mayor vínculo composicional del registro global, representando $65 \%$ de la muestra, lo cual indicaría una cierta homogeneidad composicional, más allá de las diferencias internas marcadas por los subgrupos. A más distancia se les relaciona el subgrupo E, a partir de tiestos con importante cantidad de minerales y rocas félsicas. Luego, a mayor separación se encuentra el subrupo $\mathrm{F}$, integrador de seis muestras que poseen la mayor cantidad de cuarzo en el registro. Finalmente, se enlaza el subgrupo G, con dos muestras diferenciadas del resto a partir de la escasa carga de cuarzo, la ausencia de piroxeno y vidrio volcánico, y la abundancia de biotita, microclino y plutonitas metamorfizadas.

Por otro lado, si bien estos agrupamientos no marcan una relación directa con los estándares formados previamente a partir de lupa binocular, el estudio exploratorio señala algunas tendencias interesantes. Estas se vinculan a los agrupamientos de muestras de los estándares 4 y 5 en dos subgrupos ( $\mathrm{C}$ y F), lo que podría estar indicando la mayor uniformidad de estos grupos de pastas. La discriminación señalada también se pudo reconocer en un segundo clúster exploratorio, practicado a partir de los datos de cada estándar en conjunto, y en el que se exhibe una relación estrecha entre los estándares 2 y 3 , y 6 y 7 , conformando un grupo al que luego se asocia el estándar 1, y a mayor distancia los estándares 4 y 5 (Figura 12).

\section{Discusión}

El estudio de pastas cerámicas presentado conforma un primer aporte a la comprensión de las cadenas operativas de la alfarería de Uchuquita entre los siglos III al VI de la era, esencialmente al reconocimiento de sus etapas iniciales. Estos datos cobran aún mayor importancia por la carencia en el área de antecedentes sobre el proceso de producción en un amplio rango temporal, más allá del registro tipológico y la comparación regional de estilos cerámicos, por lo que los datos generados constituyen una referencia comparativa.

Aunque para analizar la procedencia de materias primas utilizadas en la manufactura es necesario combinar diferentes estudios que revelen la química elemental y mineralogía de los sedimentos, la petrografía permite afirmar que los constituyentes en las muestras se hallan presentes en el perfil geológico de la región. Al respecto, la mineralogía y litología granítica se corresponderían con el complejo Antinaco y con el Plutón La Costa, importantes en cuarzo, feldespato potásico, plagioclasa, biotita, muscovita y litoclastos graníticos en general, gran parte de ellos metamorfizados y alterados naturalmente. Es posible que la erosión fluvial haya liberado estos componentes de los 

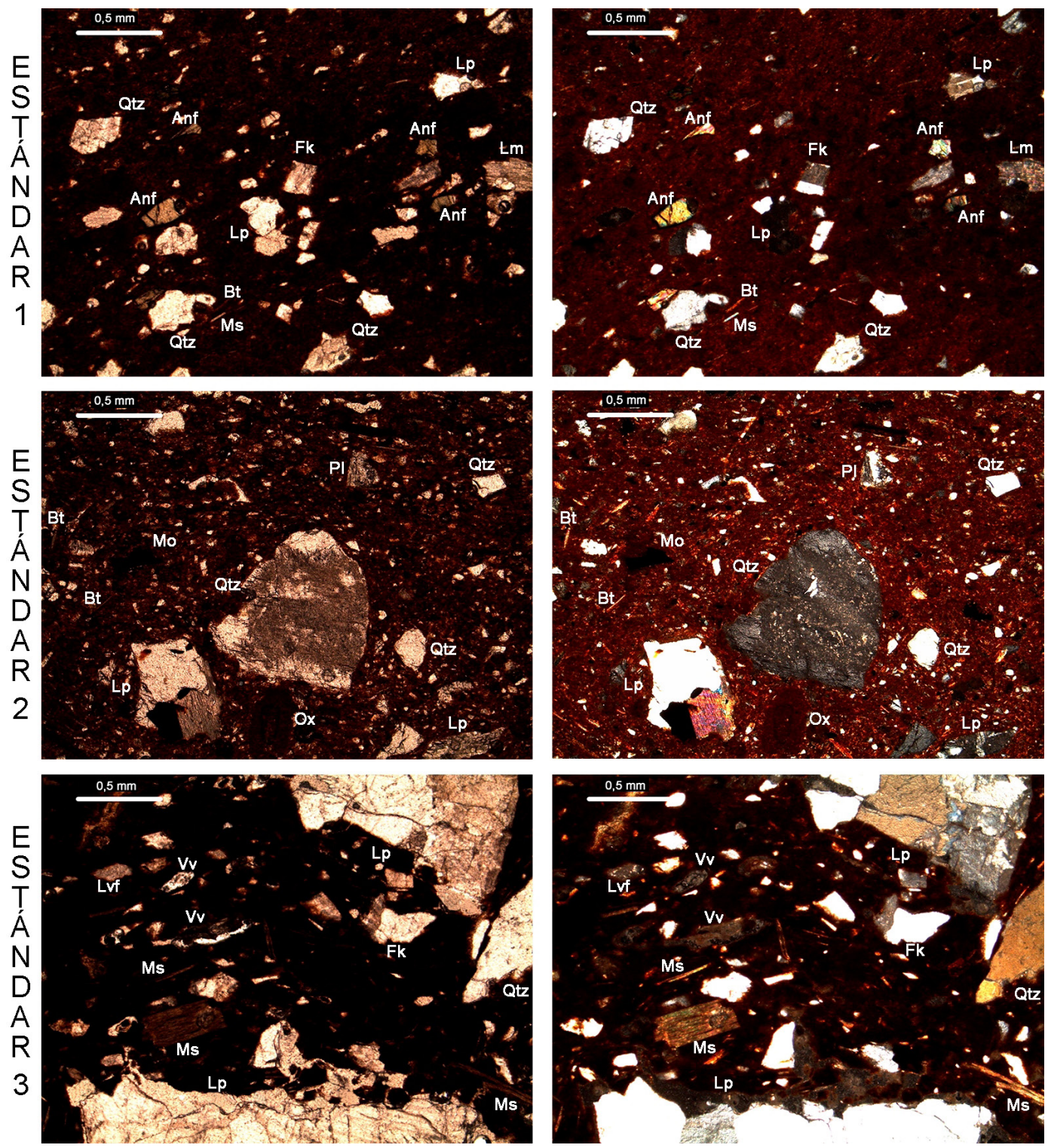

Figura 8. Microfotografías de secciones delgadas de cerámica de diferentes estándares de pasta (Izquierda: sin polarizador - Derecha: con polarizador). Estándar 1: cristales de cuarzo (Qtz), feldespato potásico (Fk), biotita (Bt), muscovita (Ms), anfíboles (Anf) y litoclastos plutónicos (Lp) y metamórficos (Lm) en matriz microgranosa/criptofilitosa. Estándar 2: cristales de cuarzo (Qtz) -algunos de ellos metamorfizados-, plagioclasa (Pl), minerales opacos (Mo), biotita (Bt), litoclastos plutónicos (Lp) y óxidos (Ox) en matriz microgranosa/pseudolepidoblástica. Estándar 3: cristales de feldespato potásico (Fk), muscovita (Ms), vidrio volcánico (Vv) y litoclastos plutónicos (Lp) en matriz microgranosa.

Thin-section photomicrographs of ceramic samples from different paste fabrics (Left: without polarizer - Right: with polarizer). Fabric 1: crystals of quartz (Qtz), potassium feldspar (Fk), biotite (Bt), muscovite (Ms), amphiboles (Anf) and plutonic (Lp) and metamorphic (Lm) lithoclasts on a micrograin/cryptophyllitose matrix. Fabric 2: crystals of quartz (Qtz) -some of them metamorphosed-, plagioclase (Pl), opaque minerals (Mo), biotite (Bt), plutonic lithoclasts (Lp) and oxides (Ox) on a micrograin/ pseudolepidoblastic matrix. Fabric 3: crystals of potassium feldspar ( Fk), muscovite (Ms), volcanic glass (Vv) and plutonic lithoclasts (Lp) on a micrograin matrix. 

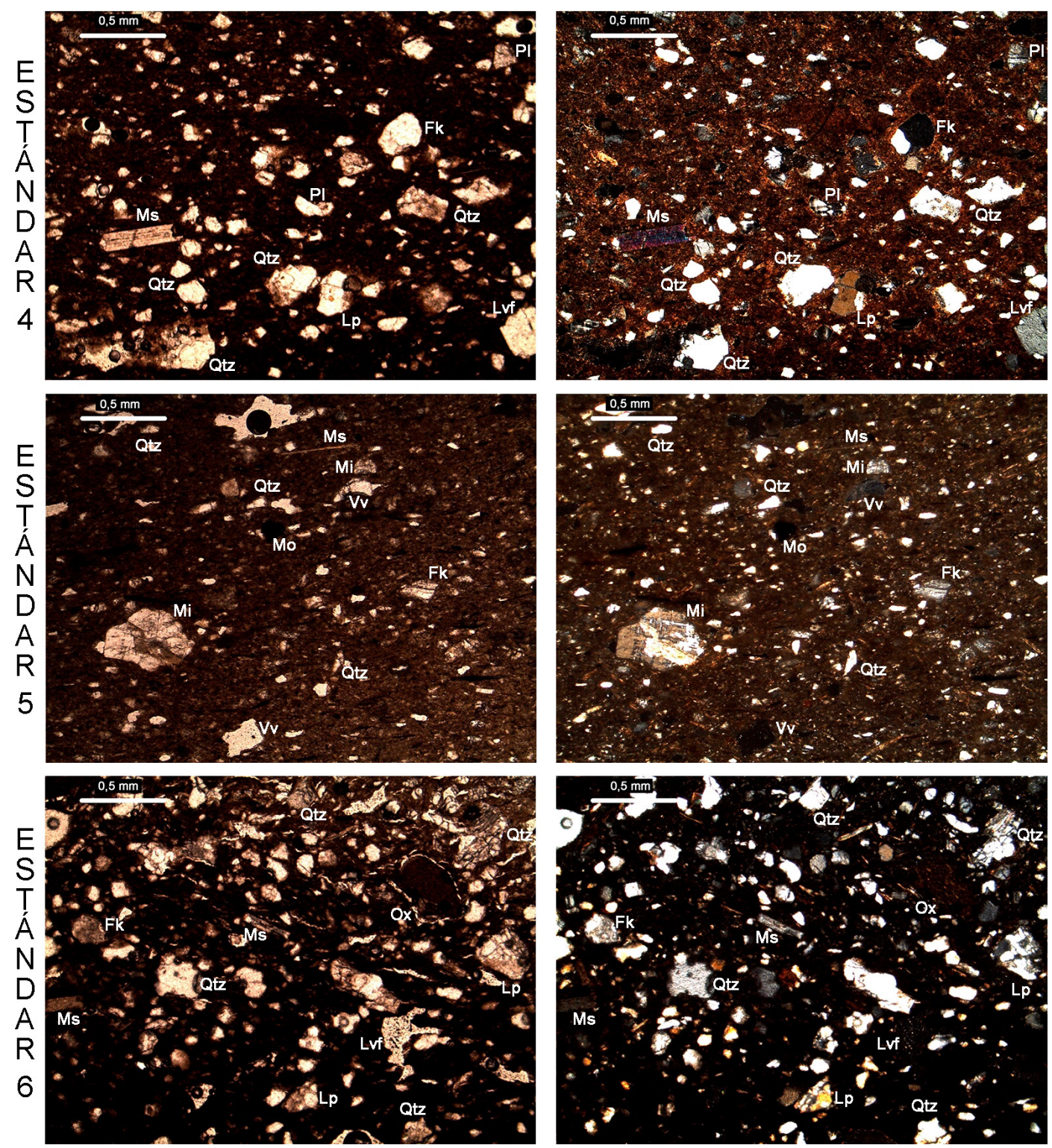

Figura 9. Microfotografías de secciones delgadas de cerámica de diferentes estándares de pasta (Izquierda: sin polarizador Derecha: con polarizador). Estándar 4: cristales de cuarzo (Qtz), feldespato potásico (Fk), plagioclasa (Pl), litoclastos plutónicos (Lp) y volcánicos félsicos (Lvf) en matriz pseudolepidoblástica. Estándar 5: cristales de cuarzo (Qtz), microclino (Mi), minerales opacos (Mo), muscovita (Ms) y feldespato potásico (Fk) en matriz microgranosa/pseudolepidoblástica. Estándar 6: cristales de cuarzo (Qtz), feldespato potásico (Fk), muscovita (Ms), óxidos (Ox) y litoclastos plutónicos (Lp) y volcánicos félsicos (Lvf) en matriz microgranosa.

Thin-section photomicrographs of ceramic samples from different paste fabrics (Left: without polarizer - Right: with polarizer). Fabric 4: crystals of quartz (Qtz), potassium feldspar ( Fk), plagioclase (Pl), and plutonic (Lp) and felsic volcanic (Lvf) lithoclastics on a pseudolepidoblastic matrix. Fabric 5: crystals of quartz (Qtz), microcline (Mi), opaque minerals (Mo), muscovite (Ms) and potassium feldspar (Fk) on a micrograin/pseudolepidoblastic matrix. Fabric 6: crystals of quartz (Qtz), potassium feldspar (Fk), muscovite (Ms), oxides $(O x)$ and plutonic ( $L p)$ and felsic volcanic (Lvf) lithoclasts on a micrograin matrix. 

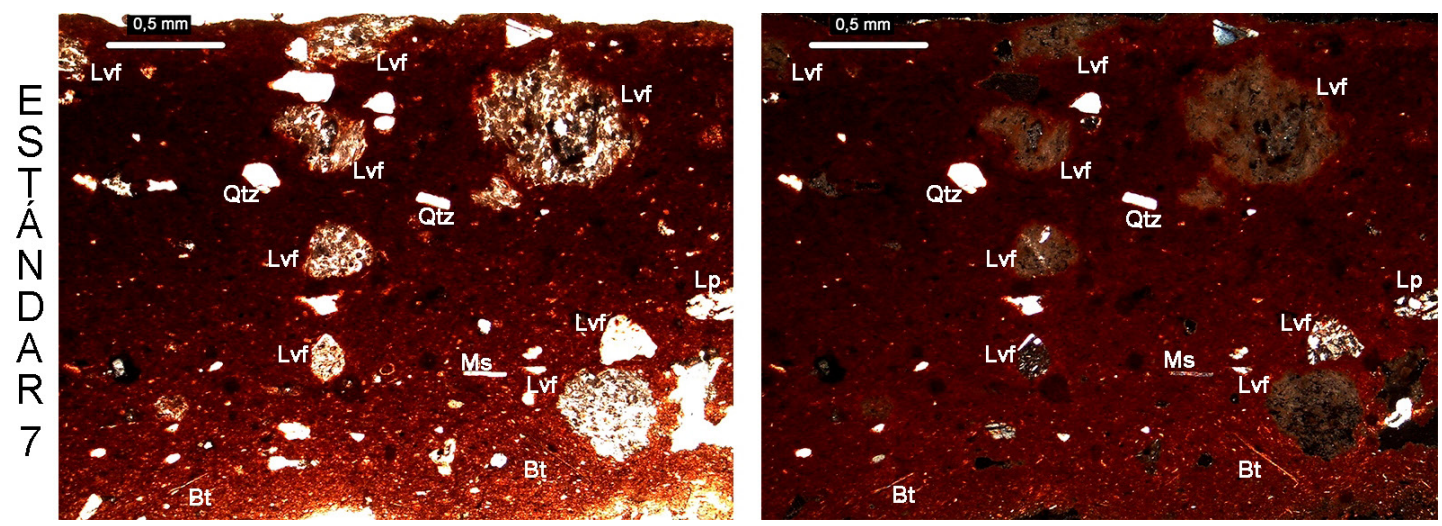

Figura 10. Microfotografía de secciones delgadas de cerámica del estándar 7 (Izquierda: sin polarizador - Derecha: con polarizador). Abundantes fragmentos de rocas volcánicas félsicas (Lvf) -vidrio volcánico alterado a cuarzo y feldespato-, cristales de cuarzo (Qtz), biotita (Bt) y litoclastos plutónicos (Lp) en matriz microgranosa/criptofilitosa.

Thin-section photomicrographs of ceramic from Fabric 7 (Left: without polarizer - Right: with polarizer). Abundant fragments of felsic volcanic rocks (Lvf) -volcanic glass altered to quartz and feldspar-, crystals of quartz (Qtz), biotite (Bt) and plutonic lithoclasts (Lp) on a micrograin/cryptophyllitose matrix.

complejos geológicos, sumándolos a la composición de los sedimentos fluviales que nacen al oeste de Uchuquita y llegan al área (González et al. 2008).

A nivel comparativo exclusivamente composicional entre los conjuntos cerámicos, el reconocimiento petrográfico y los análisis exploratorios estadísticos multivariados no proporcionaron una discriminación significativa del registro, sino más bien una homogeneidad general. Esta es determinada por la gran presencia de minerales félsicos, biotita, y granitos y granitoides metamórficos en la totalidad de las muestras. Empero, la presencia escasa de otros constituyentes observados como vulcanitas félsicas, vidrio volcánico alterado y algunas rocas volcánicas máficas podrían ser parte accesoria del material ígneo regional o quizás tener un origen más alejado del área de estudio, lo cual implicaría un probable origen alóctono de las materias primas o de algunas de las producciones. También existe la posibilidad del uso de diferentes vetas de aprovisionamiento dentro de un mismo ámbito geográfico como es el faldeo noreste de la Sierra de Velasco. Al respecto, algunos indicadores podrían estar señalando cierta distinción los materiales de algunos estilos, como por ejemplo la mayor variabilidad de estándares dentro de la alfarería Ciénaga y/o Saujil Gris, principalmente los estándares 4 y 5 . La textura de fondo de pasta del estándar 4, muy diferente del resto, también podría ser un rasgo particular sobre el uso de diferentes materiales para la manufactura, aunque también puede ser resultado de la cocción (Puente 2012). Por otro lado, el estándar 1, muy escaso en el universo cerámico de Uchuquita, posee menos relación composicional con el resto de las muestras a exhibir la mayor cantidad de granitos metamorfizados, por lo que cabe la posibilidad su origen alóctono para estos primeros momentos del primer milenio.
En definitiva, en una primera instancia de acercamiento a la procedencia de materiales para la producción de la mayoría de las cerámicas se infiere una selección local y/o micro-regional de materias primas por parte de los alfareros. Si se considera que Uchuquita poseería, por el momento, alrededor de 300 años de ocupación, los resultados revelarían una continuidad en las prácticas selectivas de materias primas y una fluida transmisión de conocimientos entre los/as ceramistas, sin cambios sustanciales en esta fase operativa. El avance de investigaciones, la identificación de minerales arcillosos así como la composición química elemental de las pastas y de arcillas, limos y arenas, permitirá corroborar esta hipótesis, además de obtener mayor certeza en el reconocimiento uniforme o no de los conjuntos alfareros, su vínculo con la geología regional, y su origen.

Desde el punto de vista tecnológico, los análisis permitieron acercarse a algunos aspectos sobre el tratamiento de pastas para los diferentes estilos, en los que se reconoce puntos de regularidad e irregularidad en las cadenas operativas. En primer lugar, la relativa uniformidad granulométrica, de distribución y densidad, y de formas de los diversos antiplásticos sugiere el empleo de sedimentos arcillosos de granulometría muy fina/fina, con escasa incorporación de inclusiones, a excepción del estándar 3 del estilo Allpatauca en el que las inclusiones se exhiben con mayor presencia. Por su parte, es factible para los estándares 1 y 5 una nula agregación de antiplásticos, lo que implicaría un tratamiento controlado y regular de los barros arcillosos (levigado, tamizado, extracción de antiplásticos mediante purificación de sedimento) en el que no queden muchos rastros de cristales de más de $0,06 \mathrm{~mm}$, o bien el uso de arcillas "limpias" con esas particularidades (Albero Santacreu y Mateu 2012; Rye 1981). Si bien el grado de angulosidad moderado que 


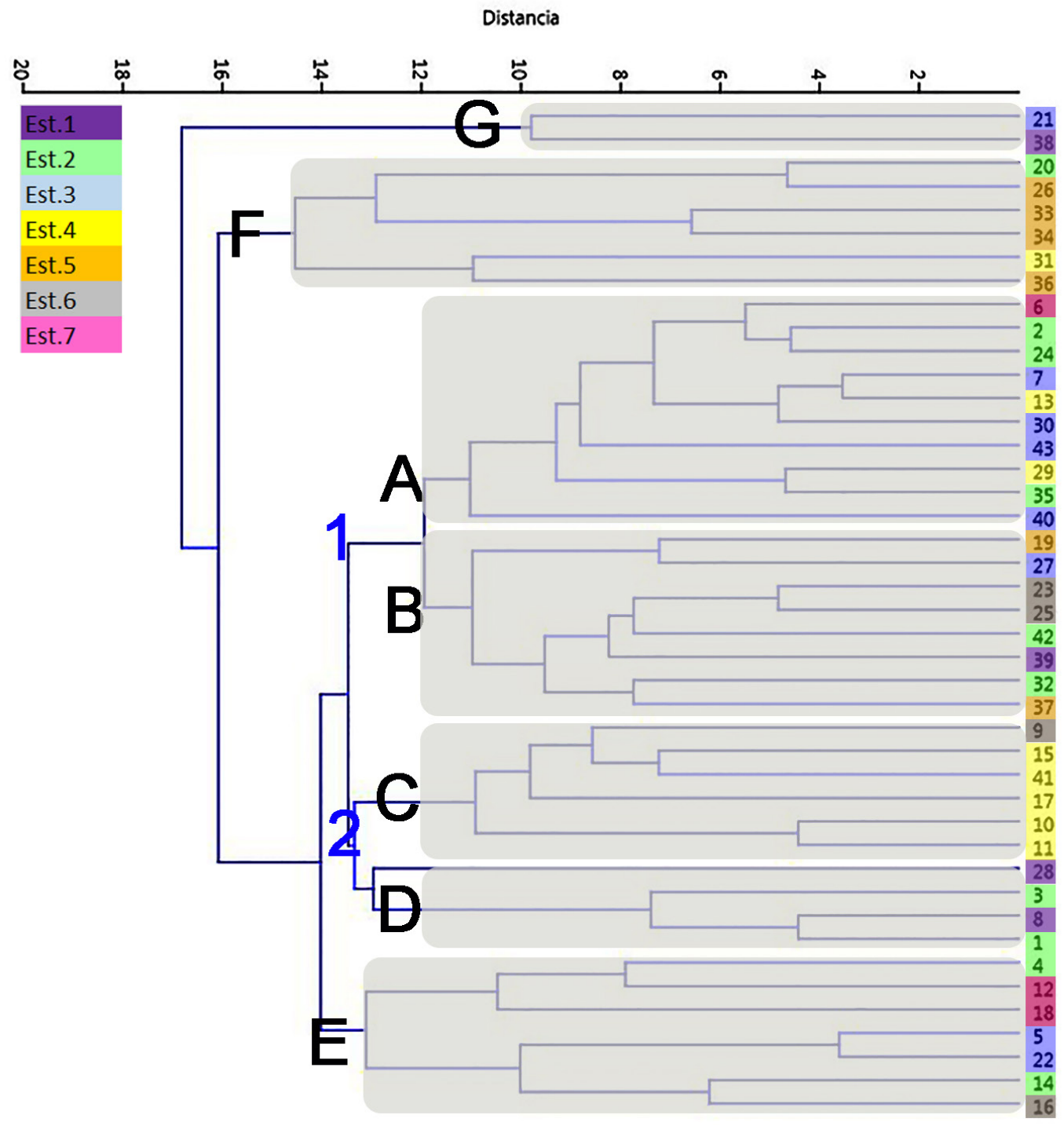

Figura 11. Dendograma exploratorio y agrupamientos de la muestra cerámica total según porcentaje y tipo de antiplásticos. Agrupamiento centroide y distancia euclidiana al cuadrado (método Warth).

Exploratory dendrogram and groupings of the total ceramic sample according to percentage and type of aplastic materials. Centroid grouping and Euclidean distance squared (Warth method).

exhiben los clastos en el resto del universo cerámico indicaría un leve tratamiento (machacado, molido) de los materiales, es probable que sea resultado de la erosión natural de las formaciones geológicas, con similares características (Alasino et al. 2006). Esta homogeneidad habría proporcionado una alta consistencia a las pastas durante el levantado y a disminuir su contracción durante el secado (Albero Santacreu 2014). En el caso de la alfarería Allpatauca, la granulometría media quizás responda a otorgar más consistencia a las vasijas generalmente de tamaño mediano y grande (cuencos y ollas globulares) (Rye 1976). Las particularidades señaladas se asemejan a las de diferentes registros de cerámicas Aguada, Ciénaga, Saujil y otros conjuntos alfareros del Formativo del centro-oeste de Catamarca y norte de La Rioja, en los que se reconoce una nula o muy escasa inclusión de antiplásticos como elección técnica para el armado de pastas (Cremonte y Pereyra Domingorena 2013:50-51 y 93; Feely et al. 2016:163; Soto et al. 2018; entre otros). 


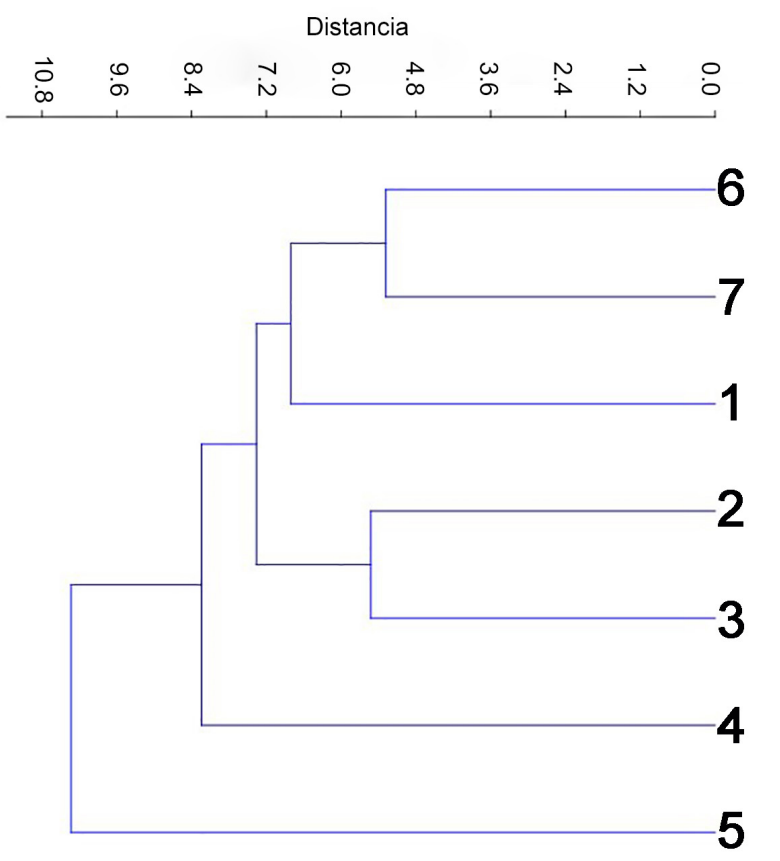

Figura 12. Dendograma exploratorio de estándares cerámicos según porcentaje y tipo de antiplásticos. Agrupamiento centroide y distancia euclidiana al cuadrado (método Warth).

Exploratory dendogram of ceramic fabrics according to percentage and type of aplastic materials. Centrid grouping and Euclidean distance squared (Warth method).

Por otro lado, la tonalidad regular naranja/rojiza y marrón/rojiza en las pastas (estándares 1, 2, 3 y 7), vinculadas a los estilos Aguada, Ciénaga Naranja y Allpatauca, sugiere cocciones en atmósferas oxidantes, lo que contrasta con los restantes estándares pertenecientes al estilo Ciénaga y/o Saujil Gris, en los que la coloración marrón/grisácea y grisácea indicaría cocciones reductoras o por lo menos una reducción en la fase final de la cocción (García Rosselló y Calvo Trías 2006). Asimismo, la ausencia de cambio de coloración en la secuencia de pasta, más la evidencia de texturas fundamentalmente compactas, dureza de categorías dura y muy dura, y fractura cortante y laminar, señalarían cocciones muy controladas, largas y estables en cada uno de los estilos (Rice 1987).

Teniendo en cuenta estos datos, el registro cerámico de Uchuquita mostraría rasgos de continuidad y ruptura en algunos aspectos tecnológicos, desde el punto de vista de la diacronía ocupacional del área. Por un lado, la homogeneidad en el tratamiento de las pastas de la mayor parte de los estándares de los estilos evidenciaría una relativa continuidad y/o sincronía en algunas "recetas de manufactura" a la hora de manipular las pastas. Dado que esta etapa de la cadena operativa tiene un carácter poco visible, difícil de modificar en el tiempo, implicaría un importante conservadurismo en la práctica, con alfareros/as imbuidos de conocimientos técnicos uniformes y bajo similares patrones de racionalidad, mantenidos independientemente de los posibles cambios producidos en otros pasos de la secuencia técnica como el levantado de formas, la decoración y la cocción (Livingstone Smith 2007; Sillar y Tite 2000). La relativa variabilidad en la tecnología de pastas registrada dentro del estilo Ciénaga y/o Saujil Gris (estándares 4, 5 y 6) podría ser un indicador de diferentes aspectos: la expresión dentro de un mismo grupo productor con distintas elecciones relacionadas al procesamiento de materias primas; tradiciones de manufactura diferentes vinculadas a múltiples comunidades regionales dentro de un mismo marco temporal; la presencia de piezas que corresponden a distintos momentos dentro de la secuencia de ocupación.

Más allá de esto, el amplio predominio de este estilo dentro del universo alfarero, y la ausencia por el momento de estructuras de combustión, desechos de manufactura y residuos cerámicos, permite plantear tentativamente la presencia de producciones de baja escala, con cierta variabilidad en la preparación de materias primas y tecnología simple, aspectos correspondientes a la expectativa de contextos alfareros 
domésticos, familiares (Costin 1990; Harbottle 1982; Rice 1987; van der Leeuw 1984). De acuerdo a estudios etnográficos (Druc 1996; Gosselain 2002; Varela Guarda 2002; entre otros), en estas sociedades es común la existencia de ceramistas con diversos grados de pericia y con instrucciones variadas, propio de sujetos encargados de múltiples tareas además de la producción, lo que se traduce muchas veces en ciertas variabilidades en diferentes atributos cerámicos, generalmente en las etapas finales de la cadena operativa (tipo de formas, tratamientos decorativos). Entendiendo que el estilo Ciénaga y/o Saujil Gris se asociaría con los primeros momentos de ocupación en el área (siglos III a VI DC), este tipo de sistema productivo es coherente con un modelo de desarrollo de comunidades sedentarias con estructura descentralizada y escasa desigualdad social (Cahiza et al. 2017).

La alfarería Allpatauca marca una diferenciación con los restantes estilos respecto al tratamiento de pastas. La mayor densidad y granulometría de antiplásticos marcaría transformaciones y/o diferencias que podrían estar ligadas al uso de diversos bancos de materias primas, a una menor preparación de los sedimentos de acuerdo a necesidades tecnológicas simplificadores del proceso de fabricación, y/o a cambios en distintos aspectos del contexto productivo, difíciles de determinar. Se trata de pocas muestras, por lo que momentáneamente no es posible avanzar en estos supuestos.

Por otra parte, las disparidades en los modos de cocción, oxidantes en los estilos Ciénaga Naranja, Allpatauca y Aguada, en contraposición con la alfarería Cienaga y/o Saujil Gris de muy probable cocción reductora, representarían diversas situaciones a profundizar, desde elecciones diferenciadas por los/as ceramistas para esta fase operativa, hasta una diversidad de bienes en Uchuquita, autóctonos y alóctonos. Ahora bien, las piezas con cocciones oxidantes son presuntamente asociadas hacia mediados y fines de la secuencia de ocupación, por lo que existe la posibilidad de ciertos cambios en el tiempo en las elecciones durante esta etapa. Investigaciones etnográficas y arqueológicas (Pool 2000; Rice 1987; Sillar 2000) asocian las trasformaciones producidas en los modos de cocción a aspectos como la introducción de nuevas tecnologías (innovaciones en las estructuras de combustión y combustibles), influencias externas, intercambios, la búsqueda de exaltar los atributos visibles de la alfarería (color), y el surgimiento de nuevos escenarios socioeconómicos que modifiquen la relación costo/beneficio al introducir cambios en el seno de la producción. Dado que en este aspecto de la cadena operativa no se posee muchos datos del contexto arqueológico ni arqueométricos, no se puede plantear por el momento una hipótesis. En cualquier caso, evidencias como las texturas, la dureza y la fractura de pasta son muy similares en los diversos estilos, por lo que las estrategias de cocción siempre fueron bien observadas y controladas.

Respecto a las funcionalidades de los conjuntos cerámicos en relación con las pastas no pareciera haber, por lo menos desde este estudio, un vínculo directo entre ellas y alguna función en particular de los recipientes (almacenamiento, consumo doméstico, festividad, etc.). Tanto las piezas decoradas como no decoradas, así como las diversas formas, poseen similar composición y tecnología, por lo que las prácticas de selección de sedimentos y su manipulación primaria no estarían sujetas a funciones particulares. Esto no implica una ausencia de funcionalidad asociada a las pastas, sino más bien que ella puede llegar a estar ligada a otros factores que se ajusten esencialmente a las consideraciones de los productores y no a las de los consumidores. Estos pueden ser diversos y asociarse a: la disponibilidad, las propiedades mecánicas, conductuales y resistencia térmica de los materiales y su idoneidad para la producción (Schiffer y Skibo 1987; Tite y Kilikoglou 2002); la reducción de los tiempos de manufactura -particularmente por el uso de barros homogéneos de granulometría fina- (Arnold 2000; Stark et al. 2000); o incluso aspectos que trascienden la esfera de la tecnología y se insertan en otros campos -económico, político e ideológico- de manera entrelazada (Calvo Trías y García Rosselló 2011).

La funcionalidad de los objetos cerámicos podría estar vinculada con otros atributos a explorar como la morfología y la decoración. Los resultados iniciales en estos rasgos (Sabatini y Garate 2017:116) establecen que las piezas decoradas y no decoradas, y las distintas formas, habrían tenido una multiplicidad de funciones. Los ceramios no evidencian marcas de exposición al fuego o algún otro tipo de desgaste indicadores de función en particular, a la vez de que se hallan comúnmente sin discriminación espacial. Sin embargo, en áreas cercanas y con una cronología un poco más tardías como El Chañarcito (siglos VI al VIII DC), la alfarería exhibiría ciertos roles de acuerdo al espacio público o doméstico (Cahiza et al. 2017). Se indica la presencia predominante de piezas decoradas y formas no restringidas (platos, cuencos) en áreas comunitarias (plataformas ceremoniales), y de piezas restringidas y no decoradas (ollas y jarras) en recintos domésticos. Para Uchuquita hasta el momento los análisis del registro arqueológico y patrón de asentamiento no precisan actividades domésticas definidas en las estructuras residenciales, sino más bien múltiples. En futuros trabajos deberán explorarse aspectos funcionales de la manufactura vinculados al levantado de vasijas, las formas y las decoraciones.

Finalmente, concibiendo a la cadena operativa alfarera como reproducción social cotidiana (Gosselain 2000), y reconociendo la homogeneidad composicional, 
se observa que las primeras fases del proceso productivo resultarían de la cercanía e interacción frecuente entre individuos insertos en una comunidad y vinculados a grupos aledaños, los cuales conocerían y compartirían fuentes de aprovisionamiento, y reproducirían similares modelos de elaboración, además de promover una construcción del paisaje y su uso (Livingstone Smith 2000; Wilshusen y Stone 1990). Los espacios de extracción de materias primas y de fabricación alfarera -en donde la cercanía residencial es significativa- favorecen no solo la relación sociocultural de los productores, sino también vínculos intratecnológicos en los que se difunden formas de elaborar cerámica y otras producciones materiales (Sillar 2000; Sofaer 2006). Asimismo, si bien hasta el momento en Uchuquita no se han hallado áreas públicas bien delimitadas, la recurrencia en las técnicas constructivas de los espacios co-residenciales y campos de cultivo materializaría un rasgo identitario comunitario (Sabatini y Salminci 2017), algo que también sucedería con la alfarería, la cual no solo jugaría un rol doméstico sino también posiblemente un papel en actividades supra-domésticas ocasionales ajenas a la cotidianidad, como celebraciones y actividades de calendario agrícola, contextos en el que se acentúan identidades y pertenencias grupales, y se ciñen vínculos espacialmente más extensos (Cahiza et al. 2017).

Estas esferas de relaciones pueden llegar a ser parte de los factores que llevan a considerar que las tradiciones de manufactura de Uchuquita compartirían ciertos atributos tecnológicos con otros registros regionales en las características de pastas cerámicas (Cremonte y Pereyra Domingorena 2013; Feely et al. 2016; entre otros), y fundamentalmente aspectos como las tonalidades, formas y decoraciones (Balesta et al. 2015; Callegari et al. 2015; entre otros), por lo que es posible que algunas decisiones técnicas estén basadas en un contexto geográfico más amplio, en donde los conocimientos, ideas y valores entrelazados juagarían un rol esencial para la construcción de una tradición manufacturera común durante los inicios del primer milenio.

\section{Conclusiones}

La alfarería es una tecnología que no solo manifiesta la necesidad de satisfacción utilitaria y adaptativa de un grupo, sino que también es una práctica sociocultural compleja, sumida a un contexto económico, político e ideológico que la determina. Implica una cultura material que refleja la forma de actuar del ceramista en vínculo estrecho con el ambiente y con otros individuos a partir de la enseñanza/aprendizaje, las costumbres, y el sistema de producción en el que surge y lo condiciona.

Se han presentado y discutido los resultados del análisis submacroscópicos y microscópicos de pastas cerámicas de Uchuquita, Anillaco. Se ha ampliado la información respecto a laidentificación y caracterización de los estilos alfareros del área. Se entiende que estos tipos de estudios son una herramienta ventajosa para conformar conjuntos de pastas en un universo cerámico, reconocer las materias primas y su tratamiento por parte de los/as alfareros/as, entender las prácticas de elaboración en su contexto sociocultural, y acercarse al conocimiento sobre el modelo de producción en un momento histórico determinado.

Los resultados permiten afirmar, hasta el momento, que las pastas de los estilos de Uchuquita forman parte de tradiciones de manufacturas muy probablemente locales, aunque también con características basadas posiblemente en producciones regionales. Esto se refleja en una homogeneidad composicional y en ciertas técnicas comunes aplicadas en el preparado de las pastas y en la diversidad en los modos de cocciones. Las elecciones dentro de las primeras fases de la cadena operativa respondieron a pautas cotidianas de enseñanza/ aprendizaje, con ideas compartidas, transferidas y heredadas desde las prácticas alfareras y los contextos de interacción social. Las decisiones de los/as ceramistas se basaron probablemente en la multiplicidad de agentes encargados, la disponibilidad ambiental, las propiedades y cualidades de los sedimentos, y el contexto sociocultural que enmarcó la producción.

Las elecciones técnicas durante la selección y tratamiento de pastas posiblemente se prolongaron en el tiempo, sin modificaciones sustanciales, mientras que aspectos como las técnicas de cocción se alternaron o modificaron de acuerdo a innovaciones tecnológicas producto de diferentes factores, en el marco de nuevos ordenamientos de los esquemas económicos y socioculturales desde mediados del primer milenio, durante el surgimiento de estructuras comunitarias locales de mayor tamaño e interacción social (ca. siglos VI-IX DC).

La identificación de estas prácticas en el pasado supone entender que el proceso de manufactura en una comunidad y en un espacio específico es muy complejo y estará condicionado por variables culturales y naturales que repercutirán en el producto final, las características particulares de la organización de la producción y los consumidores. Estos estudios, si bien conforman un importante acercamiento a la producción alfarera durante el primer milenio en Anillaco, deberán ser complementados y contrastados con un aumento de la muestra alfarera de Uchuquita y de sitios aledaños, además del estudio de fuentes de materia prima, y la combinación con otros análisis arqueométricos y convencionales. Esto beneficiará un mejor conocimiento composicional y tecnológico de los diversos estilos, así como también el de los actores involucrados y su contexto.

Agradecimientos: Al gobierno de La Rioja y del Dpto. Castro Barros, y a la comunidad de Anillaco. A quienes financiaron estos trabajos, CONICET y 
UNCUYO. A Carlos Bustamante, Sergio de La Vega y Marcos Macchioli Grande (CRILAR), a Amancay Martínez (UNSL) y a Leticia Gasparotti (CONICET-
UNCA). Finalmente, se agradece a los evaluadores, por sus correcciones y sugerencias, las cuales contribuyeron a mejorar sustancialmente este trabajo.

\section{Referencias Citadas}

Alanis, R. 1974. Material Arqueológico de la Región Diaguita. Publicaciones del Museo Regional Inca Huasi, La Rioja.

Alasino, P., J. Dahlquist, C. Galindo y C. Casquet 2006. Plutón La Costa, una expresión de magmatismo tipo-s en el sector noreste de la Sierra de Velasco, Sierras Pampeanas. Revista de la Asociación Geológica Argentina 61 (2):161-170.

Alasino, P., M. Larrovere, S. Rocher, J. Dahlquist, M. Basei, V. Memeti, S. Paterson, C. Galindo, M. Macchioli Grande y M. da Costa Campos Neto 2017. Incremental growth of an upper crustal, A-type pluton, Argentina: Evidence of a re-used magma pathway. Lithos 284-285:347-366.

Albero Santacreu, D. 2014. Materiality, Techniques and Society in Pottery Production. The Technological Study of Archaeological Ceramics through Paste Analysis. De Gruyter Open Ltd., Warsaw/Berlin.

Albero Santacreu, D. y G. Mateu 2012. Raw materials and pottery production at the Late Bronze and Iron Age Site of Puig de Sa Morisca (Mallorca, Spain). Geoarchaeology 27:285-299.

Arnold, D. 2000. Does the standarization of ceramic pastes really mean standarization? Journal of Archaeology Method and Theory 7:333-375.

Balesta, B., N. Zagorodny y F. Wynveldt 2015. El Formativo en el valle de Hualfín, una revisión crítica desde la funebria En Crónicas Materiales Precolombinas: Arqueología de los Primeros Poblados del Noroeste Argentino, editado por M Korstanje, M. Lazzari, M. Basile, F. Bugliani, V. Lema, L. Pereyra Domingorena y M. Quesada, pp. 575-602. Sociedad Argentina de Antropología, Buenos Aires.

Baxter, M. y M. Jackson 2001. Variable selection in artefact compositional studies. Archaeometry 43 (2):253-268.

Bodenbender, G. 1911. Constitución Geológica de la Parte Meridional de la provincia de La Rioja y regiones limítrofes. República Argentina. Boletín Academia Nacional de Ciencias 19 (1):1-221.

Boman, E. 1927-1932. Estudio arqueológicos riojanos. Anales del Museo Nacional de Historia Natural 35 (72):1-341.

Cabrera, A. 1976. Regiones Fitogeográficas Argentinas. Acme, Buenos Aires.

Cáceres Freyre, J. 1937. El Fuerte del Pantano. Relaciones de la Sociedad Argentina de Antropología 1:107-120.

Cahiza, P. 2015. Un acercamiento espacial a los paisajes comunitarios formativos de Los Molinos (Castro Barros, La Rioja). Relaciones de la Sociedad Argentina de Antropología 40 (1):101-122.

Cahiza, P., L. Iniesta, G. Sabatini y M.J. Ots 2017. Arquitectura y materialidad de la interacción social en la comunidad aldeana del Chañarcito, Los Molinos, La Rioja. Estudios Atacameños 57:25-44.

Calvo Trías, M. y J. García Rosselló 2011. Tradición técnica y contactos: Un marco de reflexión centrado en la producción cerámica. Rubicatum 5:1-9.
Callegari, A., M.E. Gonaldi, G. Spengler, M. Rodriguez, M. Aciar, R. Pappalardo y M. Wisnieski 2015. Tras las huellas del formativo. Norte de la provincia de La Rioja. En Crónicas Materiales Precolombinas: Arqueología de los Primeros Poblados del Noroeste Argentino, editado por M. Korstanje, M. Lazzari, M. Basile, F. Bugliani, V. Lema, L. Pereyra Domingorena y M. Quesada, pp. 247-275. Sociedad Argentina de Antropología, Buenos Aires.

Callegari, A., M.E. Gonaldi, G. Spengler y E. Aciar 2013. Construcción del paisaje en el Valle de Antinaco, departamento de Famatina provincia de la Rioja (ca. 0-1300 AD). Tradición e identidad. En Tradición e Identidad. Arqueología y Espacialidad. Enfoques, Métodos y Aplicación, editado por A. Nielsen, I. Gordillo y J.M. Vaquer, pp. 303-343. Abya Yala, Quito.

Costin, C. 1990. Craft specialization: Issues in defining, documenting and explaining the organization of production. Archaeological Method and Theory 8:1-56.

Courtois, L. 1976. Examen au Microscope Pétrographique des Céramiques Archéologiques. Centre National de la Recherche Scientifique, Paris.

Cremonte, B. 1990-1991. Análisis de Muestras de Cerámicas de la Quebrada de Humahuaca. Avances en Arqueología 1:742 .

Cremonte, B. y F. Bugliani 2006-2009. Pasta, forma e iconografía. Estrategias para el estudio de la cerámica arqueológica. Хama 19-23:239-262.

Cremonte, B. y L. Pereyra Domingorena 2013. Atlas de Pastas Cerámicas Arqueológicas. Petrografía de Estilos Alfareros del NOA. Editorial Universitaria de Jujuy, Facultad de Humanidades y Ciencias Sociales, Centro Regional de Estudios Arqueológicos, Universidad Nacional de Jujuy, San Salvador de Jujuy.

Cuomo Di Caprio, N. y S. Vaughan 1993. An experimental study in distinguishing grog (chamotte) from argillaceous inclusions in ceramic thin sections. Journal of Archaeological Science 7:21-40.

De la Fuente, G. y S. Vera 2015. Análisis tecnológicos del conjunto alfarero Saujil procedente del sitio La Florida: aportes a través de la petrografía cerámica. Libro de Resúmenes de la $1 \mathrm{ra}$ Jornada Nacional de Investigación en Cerámica, p. 10. Centro de Tecnología de Recursos Minerales y Cerámica, Buenos Aires.

Dlugosz, J., M. Gianfrancisco, A. Richard, F. Villar y V. Núñez Regueiro 2009. Arqueología del sitio El Puesto (Dpto. Castro Barros, La Rioja). Andes 20:135-160.

Dobres, M. y C. Hoffman 1994. Social agency and the dynamics of prehistoric technology. Journal of Archaeological Method and Theory 1 (3):211-258.

Druc, I. 1996. De la etnografía hacia la arqueología: aportes de entrevistas con ceramistas de Ancash (Perú) para la caracterización de la cerámica prehispánica. Bulletin de l'Institut français d'Études Andines 25 (1):17-41. 
Druc, I. 2013. What Is Local? Looking at ceramic production in the Peruvian Highlands and beyond. Journal of Anthropological Research 69 (4):485-513.

Feely, A., S. Quenardelle y N. Ratto 2016. Elecciones técnicas para la manufactura alfarera de las sociedades del primer milenio (Dpto. Tinogasta, Catamarca). Arqueología 22 (1):149-167.

García Rosselló, J. y M. Calvo Trías 2006. Análisis de las evidencias macroscópicas de cocción en la cerámica prehistórica: una propuesta para su estudio. Mayurqa 31:83-112.

Ghezzi, I. 2011. El análisis composicional en el estudio de la producción y distribución de la cerámica prehispánica. Bulletin de l'Institut Français d'Études Andines 40 (1):1-29.

Gibson,A.y A. Woods 1990. Prehistoric Pottery for the Archaeologist. Leicester University Press, Leicester.

González, A. 1998. Cultura La Aguada del Noroeste argentino (500-900 d.C.). Filmediciones Valero, Buenos Aires.

González, M., E. González Díaz, M. Elissondo, P. Juárez, M. Coppolecchia, C. Costa, H. Cisneros, G. Tello y C. Hunicken 2008. Peligrosidad sísmica en la Sierra de Velasco. Provincia de La Rioja. Peligrosidad Geológica 14, Serie Contribuciones Técnicas. SEGEMAR, La Rioja.

González de Bonaveri, M., M. Frére y P. Solá 2000. Petrografía de cerámicas arqueológicas en la cuenca del río Salado, provincia de Buenos Aires. Relaciones de la Sociedad Argentina de Antropología 25:207-226.

Gosselain, O. 1992. Technology and Style: Potters and Pottery among Bafia Cameroon. Man 27 (3):559-586.

Gosselain, O. 2000. Materializing identities: An African perspective. Journal of Archaeological Method and Theory 7 (3):187-217.

Gosselain, O. 2002. Poteries du Cameroun Meridional. Styles, Techniques et Rapports a l'Identité. Centre National de la Recherche Scientifique, Paris.

Harbottle, G. 1982. Chemical characterization in archaeology. En Contexts for Prehistoric Exchange, editado por J. Ericson y T. Earle, pp. 13-51. Academic Press, New York.

Kush, F. 1994. Investigaciones arqueológicas en la localidad de Bañados del Pantano (La Rioja). Resúmenes Expandidos del XI Congreso Nacional de Arqueología Argentina, 2da parte, p. 168. Museo de Historia Natural de San Rafael, Mendoza.

Laguens, A., M. Giesso, M. Bonnin, R.J. Speakman, M.D. Glascock, B. Manasse y N. Kriscautzky 2007. Estudio de producción y distribución de la cerámica gris-negra grabada del valle de Ambato (ca. 3000-1000 dC). En Cerámicas Arqueológicas: Perspectivas Arqueométricas para su Análisis e Interpretación, editado por B. Cremonte y N. Ratto, pp. 147-167. Editorial de la Universidad Nacional de Jujuy, Jujuy.

Lechtman, H. 1977. Style in technology - some early thoughts. En Material Culture: Styles, Organization and Dynamics of Technology, editado por H. Lechtman y R. Merril, pp. 3-20. American Ethnological Society, Minnesota.

Lemonnier, P. 1992. Elements for an Anthropology of Technology. Anthropological Papers 88. Museum of Anthropology, University of Michigan, Ann Arbor.

Leroi-Gourham, A.1964. Le Geste et la Parole I, Technique et Langage. Albin Michel, Paris.

Livingstone Smith, A. 2007. Chaîne Opératoire de la Poterie, Références Ethnographiques, Analyses et Reconstitution. Musée Royal de l'Afrique Centrale, Tervuren.
Mercado, G. 1993-1994. El yacimiento arqueológico de Anjullón. Investigación preliminar. Anales de Arqueología y Etnología 48-49:91-103.

Middleton, A., I. Freestone y M. Leese 1985. Textural analysis of ceramic thin sections: evaluation of grain sampling procedures. Archaeometry 21 (1):64-14.

Middleton, A., M. Leese y M. Cowell 1991. Computer-assisted to the grouping of ceramics fabrics. British Museum Occasional Paper 81:265-275.

Miller, H. 2007. Archaeological Approaches to Technology. Academic Press, Burlington.

Munsell Soil Color Charts 1994. Munsell Color. Macbeth Division of Kollmorgen Instruments Corporation, New York.

Nazar, D. y G. De la Fuente 2016. Acerca de la cerámica Aguada Portezuelo del valle de Catamarca y la Sierra de Ancasti. Comechingonia 20 (2):153-188.

Neff, H. 1992. Chemical Characterization of Ceramic Pastes in Archaeology. Monographs in World Archaeology 7. Prehistory Press, Wisconsin.

Olaetxea, C. 2000. La tecnología cerámica en la protohistoria vasca. Munibe 12:11-211.

Núñez Regueiro, V. y M. Tartusi 1990. Aproximación al estudio del Área Pedemontana de Sudamérica. Cuadernos del Instituto Nacional de Antropología 12:125-60.

Ortiz Malmierca, M. 2001. Loma Pircada: Estudios arqueológicos en los faldeos del Velasco. Chuquis Departamento Castro Barros, La Rioja (Argentina). Serie de Informes de Investigación Agencia Provincial de Cultura $\mathrm{N}^{\circ} 2$. Sub-Secretaría de Patrimonio Cultural y Administración de Museos Área de Arqueología, La Rioja.

Orton, C., P. Tyers y A. Vince 1997. La Cerámica en Arqueología. Crítica, Barcelona.

Pérez, J. y O. Heredia 1990. Hacia un replanteo de la cultura de La Aguada. Cuadernos del Instituto Nacional de Antropología 12:167-178.

Pool, C. 2000. Why a kiln? Firing technology in the Sierra de los Tuxtlas, Veracruz (Mexico). Archaeometry 42 (1):61-76.

Puente, V. 2012. Prácticas de producción alfarera durante el Tardío prehispánico en el Valle del Bolsón. Un aporte desde la tecnología cerámica del sitio El Duraznito (Belén, Catamarca, Argentina). Arqueología 18:101-129.

Raith, M., P. Raase y J. Reinhardt 2012. Guía para la Microscopía de Minerales en Lámina Delgada. University of Bonn, Bonn.

Ratto, N., M. Basile, A. Feely, I. Lantos, L. Coll, D. Carniglia y J.P. Miyano 2015. La gente y sus prácticas en las tierras bajas y alta del oeste tinogasteño en los siglos I a XIII D.C. (Catamarca, Argentina). En Crónicas Materiales Precolombinas. Arqueología de los Primeros Poblados del Noroeste Argentino, editado por M.A. Korstanje, M. Lazzari, M. Basile, F. Bugliani, V. Lema, L. Pereyra Domingorena y M. Quesada, pp. 215-237. Sociedad Argentina de Antropología, Buenos Aires.

Raviña, G. y A. Callegari 1991. La presencia Aguada en el departamento de Castro Barro (La Rioja). Palimpsesto 1:50-70.

Rice, P. 1987. Pottery Analysis, a Sourcebook. University of Chicago Press, Chicago.

Rye, O. 1976. Keeping your temper under control. Archaeology and Physical Anthropology in Oceania 11 (2):106-137. 
Rye, O. 1981. Pottery Technology, Principles and Reconstruction. Taraxacum, Washington D.C.

Sabatini, G. y P. Salminci 2017. Los Paisajes Aldeanos de la cuenca del río Anillaco, Castro Barros, La Rioja (ca.300800 D.C). Revista del Museo de Antropología de Córdoba, Suplemento Especial 1:7-12.

Sabatini, G. y E. Garate 2017. Espacialidad y materialidad de un conjunto de unidades domésticas tempranas -ca. 300-600 d.C. de Anillaco (La Rioja, Argentina). Comechingonia 21 (1):99-122.

Sanhueza, L. 2006. El concepto de estilo tecnológico y su aplicación a la problemática de las sociedades alfareras tempranas de Chile Central. En Puentes hacia el Pasado: Reflexiones Teóricas en Arqueología, editado por D. Jackson, D. Salazar y A. Troncoso, pp. 157-174. Serie Monográfica de la Sociedad Chilena de Arqueología, Santiago.

Schiffer, M. y J. Skibo 1987. Theory and experiment in the study of technological change. Current Anthropology 28:595-622.

Sempé, C. y M. Baldini 2011. El periodo medio en el oeste catamarqueño: Departamentos de Tinogasta y Belén. En El Hombre, el Medio y sus Relaciones, compilado por E. Silvera de Buenader y S. Martínez, pp. 218-256. Editorial Universidad Nacional de Catamarca, Catamarca.

Shanks, M. y C. Tilley 1987. Re-Constructing Archaeology:Theory and Practice. Cambridge University Press, Cambridge.

Shennan, S. 1992. Arqueología Cuantitativa. Crítica, Barcelona.

Shepard, A. 1956 [1976]. Ceramics for the Archaeologist. Carnegie Institution of Washington, Washington D.C.

Sillar, B. 2000. Dung by preference: the choice of fuel as an example of how Andean pottery production is embedded within wider technical, social, and economic practices. Archaeometry 42 (1):43-60.

Sillar, B. 2009. The social agency of things? Animism and materiality en the Andes. Cambridge Archaeological Journal 19 (3):367-377.

Sillar, B. y M. Tite 2000. The challenge of technological choices for materials science approaches in archaeology. Archaeometry $42(1): 2-20$.

Sofaer, J. 2006. Pots, houses and metals: technological relations at the Bronze Age Tell at Szazhalhalombatta, Hungary. Journal of Archaeology 25:127-147.
Soto, D., M. Rodriguez, A. Callegari y M. Del Blanco 2018. Primeras aproximaciones al estudio de las pastas cerámicas de sitios Aguada del noroeste riojano. Libro de Resúmenes Extendidos del VII Congreso Nacional de Arqueometría. Materialidad, Arqueología y Patrimonio, editado por Facultad de Ciencias Naturales e Instituto Miguel Lillo, pp. 126128. Serie Monográfica y Didáctica Volumen 56. Centro de Investigaciones en Ecología Histórica. Facultad de Ciencias Naturales e Instituto Miguel Lillo. Universidad Nacional de Tucumán, San Miguel.

Stark, M. 1999. Social dimensions of technical choice in Kalinga ceramic traditions. En Material Meanings, editado por E. Chilton, pp. 24-43. The University of Utah Press, Utah.

Stark, M., R. Bishop y E. Miksa 2000. Ceramic technology and social boundaries: cultural practices in Kalinga clay selection and use. Journal of Archaeology and Method Theory 7:295-331.

Stoltman, J. 1989. A quantitative approach to the petrographic analysis ceramic thin sections. American Antiquity 54:147160.

Tite, M. y V. Kilikoglou 2002. Dowe understand cooking pots and is there an ideal cooking pot? En Modern Trends in Scientific Studies on Ancient Ceramics, editado por V. Kilikoglou, A. Hein e Y. Maniatis, pp. 1-8. BAR International Series 1011, Archaeopress, Oxford.

Toselli, J., J. Rossi, H. Miller, M. Báez, P. Grosse, J. López y L. Bellos 2005. Las rocas graníticas y metamórficas de la sierra de Velasco. Simposio Bodenbender, editado por F. Aceñolaza, G. Aceñolaza, M. Hünicken y A. Toselli, pp. 211-220. Instituto Superior de Correlación Geológica, Serie Correlación Geológica 19. Universidad Nacional de Tucumán, Tucumán.

Urteaga, M. y L. Amundaray 2003. Estudio de la cerámica procedente del puerto romano de Irún: avance de las investigaciones. Boletín Arkeolan 11:59-104.

van der Leeuw, S. 1984. Pottery Manufacture: Some Complications for the Study of Trade. En Potsand Potters. Current Approaches in Ceramic Archaeology, editado por P. Rice, pp. 55-69. University of California, Los Ángeles.

Varela, V. 2002. Enseñanzas de alfareros toconceños: Tradición y tecnología en la cerámica. Chungara Revista de Antropología Chilena 34 (1):225-252.

Wilshusen, R. y G. Stone 1990. An ethnoarchaeological perspective on soils. World Archaeology 22:104-114.

\section{Nota}

\footnotetext{
${ }^{1}$ Las dataciones fueron calibradas con la curva IntCal13 en el software OxCal 4.3.
} 